\title{
The role of leptons in electroweak baryogenesis
}

\author{
Jordy de Vries, ${ }^{a, b}$ Marieke Postma ${ }^{c}$ and Jorinde van de Vis ${ }^{c}$ \\ ${ }^{a}$ Amherst Center for Fundamental Interactions, \\ Department of Physics, University of Massachusetts, \\ Amherst, MA 01003, U.S.A. \\ ${ }^{b}$ RIKEN BNL Research Center, Brookhaven National Laboratory, \\ Upton, NY 11973-5000, U.S.A. \\ ${ }^{c}$ Nikhef, Theory Group, \\ Science Park 105, 1098 XG, Amsterdam, The Netherlands \\ E-mail: jdevries@umass.edu, mpostma@nikhef.nl, jorindev@nikhef.nl
}

ABSTRACT: We investigate the role of leptons in electroweak baryogenesis by studying a relatively simple framework inspired by effective field theory that satisfies all Sakharov conditions. In particular, we study the effectiveness of CP-violating source terms induced by dimension-six Yukawa interactions for quarks and charged leptons. Despite the relatively small Yukawa coupling, CP-violating source terms involving taus are quite effective and can account for the observed matter-antimatter asymmetry. We obtain analytical and numerical expressions for the total baryon asymmetry, the former providing important insight into what makes lepton $\mathrm{CP}$ violation relatively effective compared to quark $\mathrm{CP}$ violation. Leptons also play an important role if the CP-violating source involves top quarks. While the tau Yukawa coupling in the Standard Model is small, it significantly enhances the baryon asymmetry by transferring the chiral asymmetry in quarks, which is washed out by strong sphalerons, to a chiral asymmetry in leptons. We conclude that leptons should not be ignored even if $\mathrm{CP}$ violation is limited to the quark sector. The role of leptons can be further increased in scenarios of new physics with additional chiral-symmetry-breaking interactions between quarks and leptons, as can happen in models with additional Higgs bosons or leptoquarks. Finally, we study CP-violating dimension-six Yukawa interactions for lighter quarks and leptons but conclude that these lead to too small baryon asymmetries.

KEYwords: Cosmology of Theories beyond the SM, Beyond Standard Model, CP violation ARXIV EPRINT: 1811.11104 


\section{Contents}

1 Introduction 1

2 Set-up and methods $\quad 4$

2.1 First-order phase transition 4

2.2 Source of CP violation 5

2.3 Experimental constraints on CP-violating dimension-six operators $\quad 7$

2.4 Transport equations $\quad 9$

2.5 Electroweak sphaleron transitions 11

$\begin{array}{lll}2.6 & \text { Efficiency of quark/lepton source } & 12\end{array}$

2.7 Additional chiral-symmetry-breaking quark-lepton interactions 13

3 Baryogenesis with a tau-lepton source $\quad 14$

$\begin{array}{ll}3.1 \text { Analytical approximation } & 14\end{array}$

$\begin{array}{ll}3.2 \text { Comparison of approximations } & 15\end{array}$

$\begin{array}{lll}3.3 & \text { Parameter dependence } & 16\end{array}$

3.4 Producing the universal baryon asymmetry with a tau source 20

4 Baryogenesis with a quark source $\quad 20$

$\begin{array}{lll}4.1 \text { Top source } & 21\end{array}$

4.2 Producing the universal baryon asymmetry with a top source 24

$\begin{array}{lll}4.3 & \text { Bottom source } & 25\end{array}$

5 Consequences of additional quark-lepton interactions $\quad 27$

$\begin{array}{llr}6 & \text { Discussion and conclusions } & 28\end{array}$

$\begin{array}{ll}\text { A Benchmark values } & 31\end{array}$

$\begin{array}{ll}\text { B Weak sphalerons } & 31\end{array}$

B.1 Two-step approach 32

B.2 One-step approach 33

C Numerical method $\quad 34$

\section{Introduction}

Understanding the prevalence of matter over antimatter in our universe is one of the great challenges in particle physics and cosmology. The baryon asymmetry can be extracted from the Planck data on the cosmic microwave background [1]

$$
Y_{B} \equiv \frac{n_{b}}{s}=(8.50 \pm 0.11) \times 10^{-11}
$$


with $n_{b}$ and $s$ the baryon number and entropy density respectively. To dynamically explain this number requires satisfying the three Sakharov conditions [2]: 1) baryon number violation, 2) charge (C) and charge-parity (CP) violation, and 3) out-of-equilibrium dynamics. The standard model (SM) only fulfills the first one - electroweak sphaleron transitions active at high temperatures violate baryon number - and physics beyond the standard model is needed to explain the baryon asymmetry in the universe.

Of the existing baryogenesis theories, electroweak baryogenesis (EWBG) is particularly interesting as it is linked to electroweak scale physics and can be tested in experiments. The (minimal) beyond-the-Standard Model (BSM) ingredients in this scenario are new sources of CP violation, and an extended scalar sector that can give rise to a first-order electroweak phase transition that provides the necessary out-of-equilibrium dynamics. Both ingredients can be probed by the large hadron collider (LHC), for instance via searches for new scalars [3-6], precision Higgs studies [7, 8], and CP-odd collider observables [9-13]. Typically, the best constraints on new sources of $\mathrm{CP}$ violation, however, come from electric dipole moment measurements [14-16]. Gravitational waves produced during a first-order electroweak phase transition may be measured by LISA or other future gravitational wave detectors $[17,18]$.

The mechanism of EWBG is, in a nutshell, as follows. The first-order electroweak phase transition proceeds via the formation of bubbles of broken Higgs vacuum, which subsequently expand to eventually fill up all of space. The quarks and leptons in the plasma collide with the walls of the expanding bubbles. If these interactions violate $\mathrm{CP}$, the transmission and reflection coefficients are different for particles and antiparticles. The net result is that an overdensity of left-handed particles over antiparticles builds up in front of the bubble wall. The $(\mathrm{B}+\mathrm{L})$-violating electroweak sphaleron transitions only act on electroweak doublets, and transform this "chiral asymmetry" into a net baryon number in front of the bubble. The produced baryons are then swept up by the expanding bubble. Inside the bubble the baryon number is preserved as the electroweak sphaleron processes are strongly suppressed in the broken vacuum. For reviews of EWBG, see for example refs. [19-22].

A large number of SM extensions that could lead to successful EWBG have been proposed. In principle, a detailed phenomenological study of each individual model is required to test the feasibility of the proposed explanation of the matter-antimatter asymmetry. To avoid such a cumbersome exercise it was proposed in ref. [15] to test EWBG, or at least a large class of EWBG models, in a single framework based on the Standard Model Effective Field Theory (SM-EFT). Unfortunately, a detailed study in ref. [16] concluded that EWBG cannot be fully studied within the SM-EFT framework. The main reason is that the SMEFT breaks down in the scalar sector, because new light degrees of freedom are necessary to obtain a strong first-order phase transition. This breakdown is communicated to the CPviolating (CPV) sector of the theory via the equation of motion of the Higgs field, which is used to construct the basis set of SM-EFT operators. The result is that there is no separation of scales and higher-dimensional operators cannot be neglected, thus invalidating the SM-EFT approach. In principle, the breakdown of EFT methods occurs in the scalar sector, which thus requires new light degrees of freedom, while the CPV sector can potentially still be described by effective operators that can now also contain the new degrees of freedom. 
In this work, we mainly focus on the CPV dynamics and avoid the issue of the firstorder phase transition by describing the bubble-wall profile in terms of a phenomenological tanh-function which provides a reasonable description of actual solutions. We describe the required additional CPV by effective dimension-six CPV operators containing SM fields only. As mentioned, in principle the CPV dynamics could arise from effective operators involving any new fields that play a role in the phase transition [23], but as these are difficult to probe in experiments we ignore such interactions for now. In particular, we focus on effective dimension-six Yukawa interactions of various quarks and leptons as these are representative for popular classes of BSM models such as multi-Higgs models. Similar studies in the literature have focussed on CPV in the top-quark sector [15, 16, 24], as the large top Yukawa coupling maximizes the CPV source term in the transport equations that describe the dynamics of the particle number densities. However, taking into account the very recent new constraint on CPV operators from an improved measurement of the electric dipole moment of the electron [25], the 'top-source' scenario gives an asymmetry that is about two orders of magnitude too small to explain the baryon asymmetry [16].

The small value of the baryon asymmetry in the top-source scenario has prompted our current study of the general features of the solutions to the transport equations, to find ways to boost the asymmetry. The reasons for the inefficiency of the top-source scenario are threefold. First, the diffusion of the chiral asymmetry into the symmetric phase is not efficient for the strongly-interacting quarks [26]. Second, EDM measurements put strong constraints on CPV in the top sector [27] such that the strength of the CPV source term is limited. And third, the washout of the produced chiral asymmetry is significant for (top) quarks, as the top Yukawa and especially the strong sphaleron interactions effectively wash out the chiral asymmetry in the symmetric phase, except for regions very close to the bubble wall $[28,29]$. These problems can potentially be overcome by looking at CPV source terms involving lighter fermions. For instance, EDM limits are less stringent for bottom quarks. While the CPV source term for bottom quarks is suppressed by the smaller Yukawa coupling, the washout rate due to Higgs interactions is suppressed accordingly. As such, the total baryon asymmetry is not a simple function of the size of the Yukawa coupling.

For leptons there can be even more advantages even though they have been neglected in many studies of EWBG. While the CPV source term is suppressed, leptons diffuse into the plasma much more easily [26], the EDM limits are less stringent for muon and tau CPV interactions [30], and the washout rate is less effective because leptons do not interact via strong sphalerons. Already in refs. [26, 31-34] the effect of leptons on EWBG was studied in a range of scenarios in which the lepton Yukawa coupling is enhanced significantly with respect to the SM value. We will show that even with the small SM Yukawa interactions, including leptons can dramatically change the baryon asymmetry in models with CPV source terms involving top quarks. In addition, we show that leptonic CPV source terms can be very efficient in producing the baryon asymmetry of the universe. Scenarios with a CPV leptonic source are also great diagnostic tools as the set of transport equations is relatively simple. We use this scenario to understand the parametric dependence of the baryon asymmetry on bubble wall parameters and the size of Yukawa couplings. This study confirms that the baryon asymmetry is not a simple function of the size of the Yukawa 
coupling. And while we focus on a particular set-up, the importance of leptons and the general mechanisms at play are more general.

As we will show, the role of leptons in EWBG depends on the effectiveness of the exchange of the chiral asymmetry between quarks and leptons. In the SM this exchange is not very efficient because of the small lepton Yukawa interactions. In various BSM models there can be more efficient mechanisms, for example via the exchange of additional scalar fields. Such mechanisms can strongly boost the baryon asymmetry if the CPV source term is located in the quark sector by transferring the chiral asymmetry into the lepton sector, where it diffuses faster and suffers from less washout. Conversely, it can suppress the baryon asymmetry if the CPV source term is located in the lepton sector. We model this phenomenon by adding effective dimension-six quark-lepton interactions; this set-up qualitatively explains features of various models studied in recent literature [33, 34]. We also comment on the effect of possible new BSM quark-quark couplings, which may likewise boost the baryon asymmetry for a source located in the quark sector, as this coupling affects and limits the washout from strong sphaleron interactions.

This paper is organized as follows. In the next section we introduce the set-up and briefly describe the first-order phase transition and bubble profile, the CPV dimension-six operators and the EDM and LHC constraints. We also list the transport equations that track the number densities of the particles in the plasma. We then identify the factors that suppress the value of the baryon asymmetry in the top-source scenario and motivate the importance of leptons. We also introduce a new top-lepton interaction. We start with a discussion of the tau-source scenario in section 3. The lepton sector almost completely decouples from the quark sector, and the baryon asymmetry can be computed analytically in this limit to good accuracy. We identify the important length scales, and discuss the physics and mechanisms at play. In section 4 we give the results for the top-source scenario. The equations to solve are more intricate, but the outcome can be understood qualitatively. We also comment on the viability of a bottom-source scenario and briefly discuss even lighter fermions. Finally, in section 5 we describe the effects of a new tau-top coupling on both the tau- and top-source scenario. We end with a discussion in section 6 .

\section{Set-up and methods}

In this section we present our set-up, and briefly review the ingredients that go into the calculation of the baryon asymmetry.

\subsection{First-order phase transition}

Sakharov's out-of-equilibrium condition is not satisfied in the SM, where the electroweak phase transition is a cross-over [35-38]. There are many BSM models that modify the Higgs sector such that the phase transition becomes first order. Well studied examples are the $\mathbb{Z}_{2}$-symmetric Higgs-singlet model [39-44] and two-Higgs doublet models [45-52]. In our previous work [16], folowing up on earlier work by [53], we argued that a firstorder electroweak phase transition cannot be described in a systematic EFT expansion and explicit light degrees of freedom must be introduced. This unfortunately prohibits a 
model-independent approach, and it is necessary to pick a specific BSM model to implement the phase transition.

Once a BSM model is chosen, one can solve the tunnelling equations of motion at the nucleation temperature $T_{N}$ to find the so-called bounce solution $\phi_{b}$ [54]. We parameterize the Higgs doublet as $H=\left(H^{+}, H^{0}\right)^{T}$ and in the bubble background $\left\langle H_{0}\right\rangle=\frac{1}{\sqrt{2}} \phi_{b}(z)$. In this work, we will use the commonly used parametrization [55]

$$
\phi_{b}(z)=\frac{v_{N}}{2}\left(1+\tanh \frac{z}{L_{w}}\right)
$$

which provides a reasonable description of the bubble-wall profile in many models. More complicated profiles can be studied in similar fashion. We do not expect our findings in this work to change significantly if more complicated profiles are applied. The ansatz assumes the planar wall approximation, in which curvature effects are neglected and all functions only depend on the distance $z$ from the center of the wall in the bubble-wall rest frame. The broken phase extends to $z \rightarrow \infty$ and the symmetric phase to $z \rightarrow-\infty$. Further, $v_{N}=v\left(T_{N}\right)$ is the vacuum expectation value of the Higgs field in the broken phase at the nucleation temperature $T_{N}$, and $L_{w}$ the bubble width. In principle, these parameters should be determined by fitting the bounce solution of a BSM model to eq. (2.1). However, our prime interest is not the phase transition itself but the comparison of different sources of $\mathrm{CP}$ violation. We work with benchmark parameters $T_{N}=88 \mathrm{GeV}, v_{N}=152 \mathrm{GeV}$, and $L_{w}=0.11 \mathrm{GeV}^{-1}$ based on an explicit solution in our earlier work [16]. For the velocity of the bubble wall we take the benchmark value $v_{w}=0.05$. We will investigate how the produced asymmetry depends on these parameters.

\subsection{Source of CP violation}

The CP-violating phase of the CKM-matrix of the Standard Model is not sufficient to explain the observed baryon asymmetry [56-59] and BSM physics should provide an extra source of $\mathrm{CP}$ violation. In this work, we describe this CP violation with effective dimensionsix operators. That is, we assume that apart from a modified scalar sector that ensures a first-order phase transition, other BSM degrees of freedom are sufficiently heavy and can be integrated out leading to effective operators. In particular, we consider the flavor-diagonal CPV dimension-six operators

$$
\mathcal{L}_{6}=-i\left[\bar{Q}_{L} \tilde{Y}_{U} \tilde{H} u_{R}+\bar{Q}_{L} \tilde{Y}_{D} H d_{R}+\bar{L}_{L} \tilde{Y}_{L} H e_{R}\right]\left(H^{\dagger} H\right)+\text { h.c. },
$$

in terms of the Higgs doublet $H$ (with $\tilde{H}^{a}=\epsilon^{a b} H^{b *}$ ), the left-handed quark and lepton $\mathrm{SU}(2)$ doublets $Q_{L}$ and $L_{L}$, and the right-handed up, down, and lepton singlets $u_{R}, d_{R}$, and $e_{R}$. We have suppressed generation indices and consider the $3 \times 3$ matrices of Wilson coefficients $\tilde{c}_{U, D, L}$ to be diagonal and real (such that the operators are purely CP violating). The extension to include flavor-changing operators can be made straightforwardly. We assume the dimension-six Yukawa couplings to be proportional to the SM Yukawa couplings $y_{f} / \sqrt{2}=m_{f} / v(v \simeq 246 \mathrm{GeV}$ is the zero-temperature vev $)$ as is the case in many BSM 
scenarios and suggested by minimal flavor violation

$$
\tilde{Y}_{U}=\operatorname{diag}\left(y_{u} \tilde{c}_{u}, y_{c} \tilde{c}_{c}, y_{t} \tilde{c}_{t}\right), \quad \tilde{Y}_{D}=\operatorname{diag}\left(y_{d} \tilde{c}_{d}, y_{s} \tilde{c}_{s}, y_{b} \tilde{c}_{b}\right), \quad \tilde{Y}_{L}=\operatorname{diag}\left(y_{e} \tilde{c}_{e}, y_{\mu} \tilde{c}_{\mu}, y_{\tau} \tilde{c}_{\tau}\right)
$$

Finally, we write

$$
\tilde{c}_{f}=\frac{s_{f}}{\Lambda_{f}^{2}}
$$

where $s_{f}= \pm 1$ is chosen to obtain a net number of baryons (rather than antibaryons) and $\Lambda_{f}$ is the associated scale of new physics where the EFT breaks down. We stress that these operators are just a subset of dimension-six SM-EFT CPV operators that can be constructed. The above operators are particularly efficient in generating a baryon asymmetry as they give rise to an effective CPV mass term during the phase transition. In addition to SM-EFT operators, in principle there can be CPV operators that include the unspecified light scalar degrees of freedom, which can only be included if we consider a specific UV completion of the scalar sector. We focus instead on the operators in eq. (2.2) as these can readily be constrained by EDM experiments.

The transport equations that track the number densities of the plasma particles are computed in the Closed Time Path (CTP) formalism using the methods in ref. [60]. During the electroweak phase transition in the presence of a bubble, the effective fermion mass is spacetime dependent. We split $m_{f}=\bar{m}_{f}\left(\phi_{b}, T\right)+m_{f, T}(T)$, with $m_{f, T}$ the usual finitetemperature mass and

$$
\bar{m}_{f}=\frac{y_{f}}{\sqrt{2}} \phi_{b}\left(1+i s_{f} \frac{\phi_{b}^{2}}{2 \Lambda_{f}^{2}}+\ldots\right),
$$

where the ellipses denote the finite-temperature corrections to the dimension-six operators in eq. (2.2), which can be neglected in the high-temperature expansion [16]. In the bubble background the phase of the mass matrix cannot be rotated away globally. The effective mass differs for left- and right-handed particles and antiparticles, and consequently they scatter differently off the bubble wall. This gives a source term in the transport equations that drives the chiral asymmetry.

There are different approaches for calculating the source term. The vev-insertion approximation (VIA) method of [60] treats the field-dependent mass eq. (2.5) as a perturbation. The source arises at first order in the derivative expansion, and depends crucially on the thermal corrections, as it is absent for vanishing thermal width of the fermion. Explicitly $^{1}$

$$
S_{f}=\frac{v_{w} N_{c}}{\pi^{2}} \operatorname{Im}\left(\bar{m}_{f}^{\prime} \bar{m}_{f}^{*}\right) J_{f}(T)=s_{f} \frac{v_{w} N_{c}}{\pi^{2}} \frac{y_{f}^{2} \phi_{b}^{3} \phi_{b}^{\prime}}{2 \Lambda_{f}^{2}} J_{f}(T)
$$

with $N_{c}$ the number of colors (we set $N_{c}=1$ if $f$ is a lepton), and $J_{f}(T)$ an integral expression that depends on the thermal masses of the right- and left-handed fermion and on their thermal widths [60]. The prime denotes derivation with respect to $z$, the distance to the center of the bubble wall. Although the higher order corrections are not explicitly known, on dimensional grounds the perturbative expansion in VIA is in terms of the

\footnotetext{
${ }^{1}$ We neglect the effects of collective plasma excitations, i.e. of the hole modes.
} 
parameter $\epsilon_{f}=\bar{m}_{f}^{2} / T^{2}$, with $\bar{m}_{f}$ the zero temperature fermion mass eq. (2.5). For the top quark $\epsilon_{t}=\mathcal{O}(1)$ and the expansion probably breaks down, whereas for all other SM fermions $\epsilon_{f} \ll 1$.

An alternative approach is the semi-classical method of [61-63], which uses the WKB expansion, and is also valid for large Yukawa couplings. The source term does not depend on the thermal corrections. The expression found in [63] (multiplied by $T^{3} / 6$ to match our conventions) is

$$
S_{f}^{\mathrm{WKB}}=\frac{c v_{w} N_{c} D_{f}}{12}\left(\left|\bar{m}_{f}\right|^{2} \theta_{f}^{\prime}\right)^{\prime \prime}
$$

with $c=\mathcal{O}(1), D_{f}$ the diffusion constant, and $\theta=\arg \left(\bar{m}_{f}\right)$. Since it is third order in the derivative expansion it is a factor $\left(L_{w} T_{N}\right)^{-2} \sim 10^{-2}$ suppressed with respect to the source in eq. (2.6), where we used the benchmark values listed in appendix A. Including all numerical factors negates this suppression: for our benchmark values we find that the semi-anytical and VIA source terms are of the same order of magnitude $S_{f}^{\mathrm{WKB}} / S_{f}=0.2-5$, with the larger values obtained for leptons (which have larger diffusion constants). For definiteness, in this paper we will use the source term in eq. (2.6) (and also the CP-conserving relaxation rate) derived in VIA for all fermions. The qualitative results on the role of the leptons in EWBG will not depend on this, and the results can straightforwardly be adapted to different source terms.

\subsection{Experimental constraints on CP-violating dimension-six operators}

The dimension-six operators in eq. (2.2) induce CPV couplings between Higgs bosons and fermions. In the SM once we perform a field redefinition to ensure real and diagonal fermion masses, the Higgs-fermion interactions become CP conserving and flavor diagonal. In the presence of the dimension-six operators, the total Higgs-fermion interactions are still flavor diagonal (by construction) but no longer CP conserving. We obtain

$$
\mathcal{L}_{h}=-\frac{m_{f}}{v} \bar{f} f h-\frac{s_{f} m_{f}}{\Lambda_{f}^{2}} \bar{f} i \gamma^{5} f\left(v h+\frac{3}{2} h^{2}+\frac{1}{2} \frac{h^{3}}{v}\right),
$$

in terms of the real fermion mass $m_{f}$ and $f$ sums over all quarks and charged leptons. EDM experiments can constrain some of the CPV $\bar{f} i \gamma^{5} t h$ interactions, depending on the fermion $f$. As we will argue, for EWBG purposes the only relevant interactions are those involving the top, bottom, and tau and we mainly discuss these. Couplings to lighter fermions are too weak to create sufficient baryon asymmetry.

The CPV couplings involving top, bottom, and tau fermions all induce a contribution to the electron EDM via two-loop Barr-Zee diagrams [30, 64, 65]. Their contributions are given by

$$
\frac{d_{e}}{e}=-\frac{8 \alpha_{e m}}{(4 \pi)^{3}} m_{e}\left[N_{c} Q_{t}^{2} g\left(x_{t}\right) \frac{s_{t}}{\Lambda_{t}^{2}}+N_{c} Q_{b}^{2} g\left(x_{b}\right) \frac{s_{b}}{\Lambda_{b}^{2}}+Q_{\tau}^{2} g\left(x_{\tau}\right) \frac{s_{\tau}}{\Lambda_{\tau}^{2}}\right]
$$

where $Q_{f}$ denotes the fermion charge in units of $e, x_{f}=m_{f}^{2} / m_{h}^{2}$, and $g\left(x_{f}\right)$ the two-loop function

$$
g\left(x_{f}\right)=\frac{x_{f}}{2} \int_{0}^{1} d x \frac{1}{x(1-x)-x_{f}} \log \left(\frac{x(1-x)}{x_{f}}\right) .
$$


Numerically we have $g\left(x_{t}\right) \simeq 1.4, g\left(x_{b}\right) \simeq 2.7 \cdot 10^{-2}, g\left(x_{\tau}\right) \simeq 7.7 \cdot 10^{-3}$, and for lighter fermions the function roughly scales as $g\left(x_{f}\right) \sim x_{f} \log x_{f}$. In these expressions we have for simplicity neglected QCD renormalization-group effects that mildly affect the constraints for quarks $[30,64,65]$.

The ACME experiment has recently improved the constraint on the electron EDM to $d_{e} \leq 1.1 \times 10^{-29} e \mathrm{~cm}$ at $90 \%$ c.l. [25]. Inserting this into the above expression shows that this sets a strong constraint on the CPV top-Higgs coupling for which we obtain $\Lambda_{t} \geq 7.1 \mathrm{TeV}$. For the bottom and charm we find $\Lambda_{b} \geq 0.49 \mathrm{TeV}$ and $\Lambda_{c} \geq 0.41 \mathrm{TeV}$ and for the tau $\Lambda_{\tau} \geq 0.16 \mathrm{TeV}$. For the lighter fermions no meaningful constraint can be set as the limit on $\Lambda_{f}$ is lower than the electroweak scale. The tau coupling can in principle also be constrained by the limit on the tau EDM. However, while the contribution to the tau EDM from CPV tau couplings is about a factor $\mathcal{O}\left(10^{6}\right)$ larger than the contribution to the eEDM, the experimental limit on the tau EDM is roughly a factor $\mathcal{O}\left(10^{11}\right)$ weaker [66] and no significant constraints are obtained. The story is similar for the CPV $\mu$-Higgs coupling and no significant constraint can be set. A CPV e-Higgs coupling, however, would lead to a large eEDM and we get a limit $\Lambda_{e} \geq 5.7 \mathrm{TeV}[67]$.

Additional constraints can be set by using experimental limits on hadronic EDMs. In this case, the analysis is more complicated and requires apart from several additional one- and two-loop diagrams also renormalization-group evolution factors and hadronic and nuclear matrix elements. A detailed study can be found in refs. [64, 68]. With conservative values of matrix elements linking $\mathrm{CP}$-odd quark-gluon operators to the neutron and $\mathrm{Hg}$ EDMs, we obtain $\Lambda_{t} \geq 0.7 \mathrm{TeV}$, which is significantly weaker than the eEDM constraints, while no significant constraints can be set on $\Lambda_{b}$. For completeness we also give the EDM constraints for lighter quarks. Using conservative values for hadronic matrix elements there is no significant constraint for $\Lambda_{s}$ and $\Lambda_{c}$, while $\Lambda_{d} \geq 1 \mathrm{TeV}$ and $\Lambda_{u} \geq 0.5 \mathrm{TeV}[64,68]$. Despite these weaker limits, we will see that EWBG is not efficient for CPV couplings involving light quarks.

The CPV fermion-Higgs couplings can also be probed at the LHC. At present, measurements of genuine $\mathrm{CP}$-odd observables are not precise enough to set meaningful constraints. However, the CPV couplings modify also CP-even observables via contributions proportional to $\tilde{c}_{f}^{2} \sim 1 / \Lambda_{f}^{4}$. For example, the $\mathrm{CPV}$ tau-Higgs coupling modifies the $h \rightarrow \tau \tau$ branching ratio signal strength

$$
\mu_{h \rightarrow \tau \tau}=\frac{\Gamma_{h \rightarrow \tau \tau}}{\Gamma_{h \rightarrow \tau \tau}^{S M}} \frac{\Gamma_{h}^{S M}}{\Gamma_{h}}=1+\frac{v^{4}}{\Lambda_{\tau}^{4}}\left(\frac{1}{1-4 x_{\tau}}\right)\left(1-\frac{m_{H} m_{\tau}^{2}}{8 \pi v^{2}} \frac{\left(1-x_{\tau}\right)^{3 / 2}}{\Gamma_{h}^{S M}}\right)
$$

where $\Gamma_{h}$ denotes the total Higgs width in presence of the CPV operators and $\Gamma_{h}^{S M} \simeq$ $4.1 \mathrm{MeV}$ the predicted SM Higgs width. $\mu_{h \rightarrow \tau \tau}$ has been measured by ATLAS, $\mu_{h \rightarrow \tau \tau}=1.09_{-0.17-0.22 .-0.11}^{+0.18+0.27+0.16}$, [69]. Adding the uncertainties in quadrature gives the constraint $\Lambda_{\tau} \gtrsim 0.3 \mathrm{TeV}$. Such limits are thus not very stringent. Similar analyses can be performed for other fermions, but in all cases the bounds on $\Lambda_{f}$ are well below $1 \mathrm{TeV}$ [64]. 


\subsection{Transport equations}

In this section we present the quantum Boltzmann transport equations for a system with CP-violating sources for top and bottom quark as well as the tau lepton, generalizing the results in refs. [60, 70]. From these equations we can compute the density of lefthanded particles. This density sources the electroweak sphaleron transition that generates a net baryon number. We denote the net number density - meaning the number density of particles minus antiparticles - of third-generation quarks by $t=n_{t_{R}}, b=n_{b_{R}}, q=$ $n_{t_{L}}+n_{b_{L}}$, the third-generation leptons by $\nu=n_{\nu_{R}}, \tau=n_{\tau_{R}}, l=n_{\nu_{L}}+n_{\tau_{L}}$, and similarly for the lighter generations; the Higgs number density is given by $h=n_{H^{0}}+n_{H^{+}}$.

A careful analysis of the relevant timescales for the creation of the chiral asymmetry is given in section 3. Gauge interactions and Higgs self-interactions are fast compared to the relevant time scales and are therefore assumed to be in thermal equilibrium, implying that the chemical potentials of the up and down components of $\mathrm{SU}(2)_{L}$ doublets are equal. The same holds for the components of the Higgs doublet. First- and second-generation Yukawa interactions are slow and are therefore neglected; we justify this approximation in this work. Consequently, the light leptons effectively decouple. The light quarks still participate in strong sphaleron interactions, which means their densities are related via

$$
q_{1}=q_{2}=-2 u=-2 d=-2 s=-2 c,
$$

and we only require one equation to describe them. We choose the $u$-quark. Here $q_{i}$ denotes the first- and second-generation left-handed doublet, and $u, d, s, c$ the right-handed quarks. If we neglect the bottom Yukawa interactions, we have the further simplification $u=b$.

Weak sphaleron processes are also slow and baryogenesis can be modeled as a twostep process, where in the first step a chiral asymmetry is generated, which in a second step is converted into a baryon asymmetry [63, 71]. We argue in section 3 that this twostep approach even works for a lepton source scenario, where the relevant tau-Yukawa interaction rate can be small compared to the weak sphaleron rate.

With the above considerations the full set of transport equations becomes

$$
\begin{aligned}
& \partial_{\mu} q^{\mu}=+\Gamma_{M}^{(t)} \mu_{M}^{(t)}+\Gamma_{M}^{(b)} \mu_{M}^{(b)}+\Gamma_{Y}^{(t)} \mu_{Y}^{(t)}+\Gamma_{Y}^{(b)} \mu_{Y}^{(b)}-2 \Gamma_{\mathrm{ss}} \mu_{\mathrm{ss}}+\Gamma_{\mathrm{QL}} \mu_{\mathrm{QL}}-S_{t}-S_{b}, \\
& \partial_{\mu} t^{\mu}=-\Gamma_{M}^{(t)} \mu_{M}^{(t)}-\Gamma_{Y}^{(t)} \mu_{Y}^{(t)}+\Gamma_{\mathrm{ss}} \mu_{\mathrm{ss}}-\Gamma_{\mathrm{QL}} \mu_{\mathrm{QL}}+S_{t}, \\
& \partial_{\mu} b^{\mu}=-\Gamma_{M}^{(b)} \mu_{M}^{(b)}-\Gamma_{Y}^{(b)} \mu_{Y}^{(b)}+\Gamma_{\mathrm{ss}} \mu_{\mathrm{ss}}+S_{b} \\
& \partial_{\mu} l^{\mu}=+\Gamma_{M}^{(\tau)} \mu_{M}^{(\tau)}+\Gamma_{Y}^{(\tau)} \mu_{Y}^{(\tau)}-\Gamma_{\mathrm{QL}} \mu_{\mathrm{QL}}-S_{\tau}, \\
& \partial_{\mu} \nu^{\mu}=0 \\
& \partial_{\mu} \tau^{\mu}=-\Gamma_{M}^{(\tau)} \mu_{M}^{(\tau)}-\Gamma_{Y}^{(\tau)} \mu_{Y}^{(\tau)}+\Gamma_{\mathrm{QL}} \mu_{\mathrm{QL}}+S_{\tau} \\
& \partial_{\mu} h^{\mu}=+\Gamma_{Y}^{(t)} \mu_{Y}^{(t)}-\Gamma_{Y}^{(b)} \mu_{Y}^{(b)}+\Gamma_{Y}^{(c)} \mu_{Y}^{(c)}-\Gamma_{Y}^{(\tau)} \mu_{Y}^{(\tau)} \\
& \partial_{\mu} u^{\mu}=+\Gamma_{\mathrm{ss}} \mu_{\mathrm{ss}} .
\end{aligned}
$$


In principle, we should also add a transport equation for the new light degrees of freedom added to the scalar sector. Generically, these new degrees of freedom equilibrate with the SM Higgs as significant interactions are required for a first-order phase transition, and the scalar degrees of freedom can be added to $h$ which now denotes a combined number density.

The $S_{f}$ denote flavor-diagonal CPV sources for third-generation fermions. The relaxation rates $\Gamma_{M}^{(f)}$, Yukawa rates $\Gamma_{Y}^{(f)}$, and strong sphaleron rate $\Gamma_{\mathrm{ss}}$, redistribute and/or wash out the generated chiral asymmetry. If the new degrees of freedom also couple to SM fermions additional interaction rates can appear in the above equations. We model one such possible term using $\Gamma_{\mathrm{QL}}$ that corresponds to a new top-tau interaction. This coupling will be motivated in section 2.6. The lepton sector mirrors the quark sector in the above equations, with the important difference that the leptons do not have strong sphaleron transitions, and the right-handed neutrino decouples. The values of the interaction rates for our benchmark model are given in table 5 in appendix A.

The chemical potentials (strictly speaking, these are rescaled chemical potentials, as we have factored out a factor $6 / T^{2}$ ) corresponding to the interaction rates $\Gamma_{M}^{(f)}, \Gamma_{Y}^{(f)}, \Gamma_{\mathrm{ss}}$ and $\Gamma_{\mathrm{QL}}$ are

$$
\begin{aligned}
\mu_{M}^{(t)} & =\left(\frac{t}{k_{t}}-\frac{q}{k_{q}}\right), & \mu_{Y}^{(t)} & =\left(\frac{t}{k_{t}}-\frac{q}{k_{q}}-\frac{h}{k_{h}}\right), \\
\mu_{M}^{(b)} & =\left(\frac{b}{k_{b}}-\frac{q}{k_{q}}\right), & \mu_{Y}^{(b)} & =\left(\frac{b}{k_{b}}-\frac{q}{k_{q}}+\frac{h}{k_{h}}\right), \\
\mu_{M}^{(\tau)} & =\left(\frac{\tau}{k_{\tau}}-\frac{l}{k_{l}}\right), & \mu_{Y}^{(\tau)} & =\left(\frac{\tau}{k_{\tau}}-\frac{l}{k_{l}}+\frac{h}{k_{h}}\right), \\
\mu_{\mathrm{SS}} & =\sum_{i=1}^{3}\left(\frac{2 q_{i}}{k_{q_{i}}}-\frac{u_{i}}{k_{u_{i}}}-\frac{d_{i}}{k_{d_{i}}}\right), & \mu_{\mathrm{QL}} & =\left(\frac{l}{k_{l}}-\frac{\tau}{k_{\tau}}-\frac{q}{k_{q}}+\frac{t}{k_{t}}\right) .
\end{aligned}
$$

The $k_{i}\left(m_{i} / T\right)$-functions relating the chemical potentials to the number densities are defined via $n_{i}=T^{2} \mu_{i} k_{i} / 6+\mathcal{O}\left(\mu_{i}^{3}\right)$.

In the diffusion and planar-wall approximation for the bubble profile, we can write the left-hand-side of the transport equations as [72]

$$
\partial_{\mu} n_{f}^{\mu}=v_{w} n_{f}^{\prime}-D_{f} n_{f}^{\prime \prime},
$$

with $v_{w}$ the bubble-wall speed and $D_{f}$ the diffusion coefficient (listed in appendix A). Since the left- and right-handed quarks have approximately equal diffusion constants, baryon number is locally conserved on the time scale of the transport equations such that

$$
t+b+q+c+s+q_{2}+u+d+q_{1}=t+b+q=0 .
$$

In the second expression we used that the light quarks are only produced via strong sphalerons eq. (2.12). Local baryon number conservation can be used to eliminate the transport equation for the bottom quark. The set of transport equations can be further simplified by neglecting the slower rates, but which rates can be neglected depends on the chosen source term. If bottom Yukawa interactions are neglected we have $b=u$, and the 
number densities of all quarks directly follow from $(q, t)$. If bottom Yukawa interactions are included we additionally have to solve the $u$-equation.

In contrast, lepton number is only conserved globally [32]. Right-handed leptons diffuse more easily than left-handed leptons since right-handed leptons do not interact through $S U_{L}(2)$-interactions, and therefore $D_{l} \neq D_{\tau}$. As we will see, for our set-up it is a reasonably good approximation to neglect this difference and assume local lepton number conservation as well.

The complete expressions for the interaction rates, source terms, masses, and other constants entering the equations can be found in ref. [16]. For the Yukawa rates we used the expressions in ref. [31]. The rate $\Gamma_{\mathrm{QL}}$ is discussed in section 2.7. We have solved the transport equations numerically, with the boundary condition that the number densities vanish far away from the bubble wall. Details are given in appendix C. As an extra check we also solved the equations using the semi-analytical method of ref. [73]. This method approximates all rates by a step function, and ignores the variation over the bubble-wall width. In addition, since the source peaks in the broken phase, it is set to zero in the symmetric phase. Both approximations are reasonable, and for generic input parameters we find that the numerical and semi-analytical results only differ by $\mathcal{O}(10 \%)$. Unless otherwise stated, the results presented in the upcoming sections are those of the numerical calculation.

\subsection{Electroweak sphaleron transitions}

The electroweak sphalerons convert the chiral asymmetry into a baryon asymmetry. The corresponding rate is slower than all other relevant interaction rates and thus decouples from the transport equations. The only exception is the lepton Yukawa rate and we discuss this case in section 3. The density of left-handed fermions that sources the electroweak sphaleron transitions is given by $n_{L}=\sum_{i}\left(q_{i}+l_{i}\right)$, and is determined by solving the transport equations in eq. (2.13). The baryon asymmetry becomes (see appendix B for a derivation)

$$
Y_{B}=\frac{n_{b}}{s}=-\frac{3 \Gamma_{\mathrm{ws}}}{2 s D_{q} \alpha_{+}} \int_{-\infty}^{0} \mathrm{~d} z n_{L} \mathrm{e}^{-\alpha_{-} z}
$$

where

$$
\alpha_{ \pm}=\frac{v_{w} \pm \sqrt{4 D_{q} \Gamma_{\mathrm{ws}} \mathcal{R}+v_{w}^{2}}}{2 D_{q}} .
$$

Here $s=2 \pi^{2} /(45) g_{* S} T^{3}$ is the entropy density, $g_{* S}=106.75$ the entropy degrees of freedom at the electroweak scale, $D_{q} \simeq 6 / T$ the quark diffusion constant, $\mathcal{R}=15 / 4$ the SM relaxation term, and $\Gamma_{\mathrm{ws}}=6 \kappa \alpha_{w}^{5} T$ the electroweak sphaleron rate with $\kappa \sim 20$ and $\alpha_{w}=$ $g^{2} /(4 \pi)[74-76]$. In the limit $4 D_{q} \Gamma_{\mathrm{ws}} \mathcal{R} \ll v_{w}^{2}$, the result reduces to a more familiar form

$$
Y_{B}=\frac{n_{b}}{s}=-\frac{3 \Gamma_{\mathrm{ws}}}{2 v_{w} s} \int_{-\infty}^{0} n_{L}(z) e^{z \mathcal{R} \Gamma_{\mathrm{ws}} / v_{w}}
$$

This approximation works well for $v_{w} \geq 0.02$. We integrate the asymmetry over the broken phase ranging from $z=-\infty$ to the center of the bubble wall at $z=0$ where $\phi_{b}=v_{N} / 2$. Other integration regions can be chosen, for instance $-\infty<z<-L_{w}[32,60]$. 
This gives a percent-level difference for the asymmetry generated by a lepton source, but can give an $\mathcal{O}(1)$ difference for the top source. It would be optimal to integrate over the full region using the field-dependent $\Gamma_{\mathrm{ws}}\left(\phi_{b}\right)$, but this requires a better understanding of the electroweak sphaleron rate.

\subsection{Efficiency of quark/lepton source}

A priori, one would expect the top source to give the largest baryon asymmetry. Since the CPV source term of eq. (2.6) is proportional to the Yukawa coupling squared, it is maximal for the top quark. Even if we had not assumed the dimension-six couplings to be proportional to $y_{f}$, the source would have a linear dependence on the Yukawa coupling. A first reason why the top-source scenario might nevertheless not be the most efficient mechanism to generate the BAU is also immediately apparent from eq. (2.6). The CPV source is suppressed by the square of the scale $\Lambda_{f}$. In section 2.3 we showed that the experimental bounds on $\Lambda_{f}$ are rather strong for the top quark: $\Lambda_{t} \gtrsim 7.1 \mathrm{TeV}$. For the bottom quark and the tau lepton the bounds are much less severe: $\Lambda_{b} \gtrsim 0.5 \mathrm{TeV}$ and $\Lambda_{\tau} \gtrsim 0.3 \mathrm{TeV}$ respectively.

A second reason why leptons could be more efficient than quarks in generating the BAU is their larger diffusion coefficient. Since leptons only interact via the electroweak force they can diffuse further into the symmetric phase than the strongly interacting quarks [26]. This enhances the baryon asymmetry, as the electroweak sphalerons have more time to convert the lepton asymmetry into a baryon asymmetry before the bubble wall passes.

Finally, washout effects are also maximal for top quarks. The interactions mediated by the $\mathrm{CP}$ conserving part of the mass matrix relax the chiral asymmetry; the relaxation rate for this process is proportional to $\Gamma_{M}^{(f)} \propto\left|\bar{m}_{f}\right|^{2}=y_{f}^{2} \phi_{b}^{2}+\mathcal{O}\left(\Lambda_{f}^{-4}\right)$, and thus largest for the top quark. The Yukawa-type interactions are kinematically forbidden in the plasma, but phase space opens up if an additional gluon or weak boson is radiated; the rate for this process is likewise maximized for the top quark $\Gamma_{Y}^{(f)} \propto y_{f}^{2}$.

More importantly, the strong sphaleron transitions are approximately in equilibrium in the symmetric phase except for regions close to the bubble wall $[28,29,77]$ - we will estimate the size of this region in section 4.1 - and they very effectively wash out the chiral asymmetry in the quark sector, but leave leptons untouched. Indeed, if the strong sphaleron interactions are in thermal equilibrium the corresponding chemical potential eq. (2.14) vanishes $\mu^{s s} \simeq 0$. Neglecting the Yukawa interactions of the first- and secondgeneration quarks, and using baryon number conservation gives the relation

$$
-4 u a_{1}+q a_{2}+b a_{3} \simeq 0,
$$

where

$$
a_{1}=\frac{1}{2}\left(\frac{4}{k_{q_{1}}}+\frac{1}{k_{u}}+\frac{1}{k_{d}}\right), \quad a_{2}=\left(\frac{2}{k_{q}}+\frac{1}{k_{t}}\right), \quad a_{3}=\left(\frac{1}{k_{t}}-\frac{1}{k_{b}}\right) .
$$

The chiral asymmetry in quarks becomes

$$
n_{L}^{(q)} \equiv \sum_{i=1}^{3} q_{i}=q-4 u=q\left(1-\frac{a_{2}}{a_{1}}\right)-b\left(\frac{a_{3}}{a_{1}}\right),
$$


where in the second and third steps we used eq. (2.12) and eq. (2.20), respectively. At zero temperature $\left(a_{1}, a_{2}, a_{3}\right) \propto(1,1,0)$ and the chiral asymmetry in quarks vanishes. At finite temperature there are corrections. For our benchmark point we find $n_{L}^{(q)} \simeq-0.01 q-0.01 b$, and thus the chiral asymmetry in quarks is suppressed by roughly two orders of magnitude with respect to the individual quark densities.

The above reasons ensure that the tau-source scenario is more effective at producing the baryon asymmetry than the top-source scenario. That is, despite the Yukawa suppression of the tau source we will obtain similar-sized total baryon asymmetries for $\Lambda_{t} \simeq \Lambda_{\tau}$.

The top Yukawa and especially the strong sphaleron interactions are very effective in washing out the chiral asymmetry in quarks. Hence, if some of the chiral asymmetry can be transferred to the leptons, which will escape the washout, the baryon asymmetry is increased. Leptons produced via the relatively small tau Yukawa interactions give the dominant contribution to the baryon asymmetry in the top-source scenario leading to a larger asymmetry up to an order of magnitude (see figure 3 ). The authors of refs. [26, 32, 78] already pointed out that the contribution of tau leptons can be significant in the context of a two Higgs doublet model and the MSSM where the value of $y_{\tau}$ can be boosted by a large $\tan \beta$, but we stress that this Yukawa enhancement is not required for leptons to be very relevant. This relevance can be even enhanced in models with additional chiralsymmetry-breaking lepton-quark interactions, for instance via the exchange of new scalars. We discuss this in the next section.

\subsection{Additional chiral-symmetry-breaking quark-lepton interactions}

The importance of the leptons in the top-source case becomes even more pronounced in models with additional chiral-symmetry-breaking quark-lepton interactions. In our set-up we have added a dimension-six tau-top interaction to study this effect, but the qualitative results are insensitive to the exact implementation. The dimension-six operator is given by

$$
\mathcal{L}_{\mathrm{QL}}=\frac{1}{\Lambda_{\mathrm{QL}}^{2}} \bar{\tau}_{L} \tau_{R} \bar{t}_{R} t_{L}+\text { h.c. }
$$

Using the methods of ref. [31] we have calculated the rate associated to this interaction and obtain $\Gamma_{\mathrm{QL}}=\kappa_{\mathrm{QL}} T^{5} / \Lambda_{\mathrm{QL}}^{4}$ with $\kappa_{\mathrm{QL}}$ a factor of $\mathcal{O}(1)$. This interaction becomes important if it exceeds the SM tau Yukawa rate, which, for our benchmark point, is the case for $\Lambda_{\mathrm{QL}} \lesssim 3 \mathrm{TeV}$.

For definiteness, we focus here on a new top-tau coupling but stress that a coupling to the muon or electron has a similar effect. If the CPV source is located in the quark sector, the baryon asymmetry can also be boosted by including a new coupling of the top to one of the lighter quarks (e.g. a top-charm coupling). In such a scenario the washout by strong sphalerons becomes less effective. Indeed, eq. (2.22) above was derived under the assumption that the light quarks are only produced via strong sphalerons and that their Yukawa interactions are negligible, such that eq. (2.12) holds. If, say, the new top-charm interaction is stronger than the strong sphaleron interactions, this is no longer the case, and the washout by strong sphalerons no longer implies a washout of the chiral asymmetry. 
We describe the top-tau transfer here with an effective interaction, but larger interaction rates are possible if the coupling is via exchange of a light degree of freedom, for example the new scalar particle added to get a first-order phase transition [33, 34]. In such cases the scattering can be resonantly enhanced. To properly describe this requires the inclusion of the transport equation for the light particle with the corresponding interaction rates derived from renormalizable interactions. We expect that our effective interaction gives qualitatively similar results, even in the regime where $\Lambda_{\mathrm{QL}}$ is fairly small, and we therefore treat $\Lambda_{\mathrm{QL}}$ as a phenomenological parameter. We will show in section 5 that such interactions, even of modest strength, can drastically increase or reduce the baryon asymmetry depending on the nature of the CPV source.

\section{Baryogenesis with a tau-lepton source}

We start with a discussion of baryogenesis from a CPV tau source, and assume there is no new lepton-quark coupling (that is, we set $\Lambda_{\mathrm{QL}} \rightarrow \infty$ ). The value of the tau Yukawa coupling at the electroweak scale is small, $y_{\tau} \simeq 0.01$, with respect to the top Yukawa coupling, $y_{t} \simeq 1$. For reasons discussed in section 2.6, the tau could nevertheless be an interesting source of the baryon asymmetry.

In the present scenario, the lepton sector essentially decouples from the quark sector and the baryon asymmetry can be computed analytically to good accuracy. We start with discussing the analytical approximation and later compare it to the full numerical solution that does include effects of the quark sector. The analytical solution clarifies the dependence of the baryon asymmetry on parameters associated to the bubble wall and the leptonic Yukawa couplings. This relatively simple set-up provides insight into more complicated scenarios where analytical solutions are not possible.

\subsection{Analytical approximation}

The transport equations in eq. (2.13) contain separate equations for the third-generation left-handed doublet, $l^{\mu}$, and the right-handed singlet, $\tau$. Because the left- and right-handed tau leptons diffuse at different rates: $D_{l}=100 / T$ and $D_{\tau}=380 / T$ [32], in principle we have to treat these number densities separately. However, to a reasonable approximation we can ignore this difference and set $D_{l}=D_{\tau}=100 / T$, which implies $l=-\tau$. The lepton transport equation simply becomes

$$
-D_{l} l^{\prime \prime}+v_{w} l^{\prime}+\bar{\Gamma} l-\Gamma_{Y}^{(l)} \frac{h}{k_{h}}=-S_{\tau}, \quad \text { with } \quad \bar{\Gamma}=\left(\Gamma_{M}^{(l)}+\Gamma_{Y}^{(l)}\right)\left(\frac{1}{k_{\tau}}+\frac{1}{k_{l}}\right) .
$$

CPV resides in the lepton sector and any non-zero density of Higgs particles can only be generated via interactions with leptons. Since the lepton Yukawa rate is relatively slow the Higgs density remains small and we can approximate $h \simeq 0$. In this limit, the leptons decouple completely and to find the chiral asymmetry we only have to solve eq. (3.1). This can be done using the semi-analytical solution mentioned at the end of section 2.4, which gives

$$
l=-\frac{2 \bar{S}}{\left(\sqrt{4 D_{l} \bar{\Gamma}_{B}+v_{w}^{2}}+\sqrt{4 D_{l} \bar{\Gamma}_{S}+v_{w}^{2}}\right)} \mathrm{e}^{z / L_{S}}, \quad \text { for } z<0,
$$


with $\bar{\Gamma}_{\{S, B\}}$ the rescaled interaction rates eq. (3.1) in the symmetric and broken phase respectively, and

$$
\begin{aligned}
L_{i} & =\frac{2 D_{l}}{\left(v_{w}+\sqrt{4 D_{l} \bar{\Gamma}_{i}+v_{w}^{2}}\right)}, \\
\bar{S} & =\int_{0}^{\infty} \mathrm{e}^{-y / L_{B}} S_{\tau}(y) \mathrm{d} y=\frac{15 s_{\tau}}{128 \pi^{2}} \frac{y_{\tau}^{2}}{\Lambda_{\tau}^{2}} v_{w} v_{N}^{4} J_{\tau}(T)\left[1-\frac{4(7+6 \ln 2)}{45} \frac{L_{w}}{L_{B}}+\mathcal{O}\left(\frac{L_{w}^{2}}{L_{B}^{2}}\right)\right],
\end{aligned}
$$

and $i=\{S, B\}$. To get the second expression for $\bar{S}$, we used eqs. (2.1) and (2.6), and expanded in $L_{w} / L_{B}$. For a tau source, the leading order term dominates by far and we can set $L_{w} / L_{B} \simeq 0$.

Integrating the chiral asymmetry, $n_{L}=l$, as in eq. (2.19) gives the baryon asymmetry

$$
Y_{B}=\frac{3 \Gamma_{\mathrm{ws}}}{2 v_{w} s} \frac{2 \bar{S}}{\left(\sqrt{4 D_{l} \bar{\Gamma}_{B}+v_{w}^{2}}+\sqrt{4 D_{l} \bar{\Gamma}_{S}+v_{w}^{2}}\right)} \frac{L_{\mathrm{ws}} L_{S}}{L_{S}+\frac{1}{2} L_{\mathrm{ws}}\left(1+\sqrt{1+\frac{4 D_{q}}{L_{\mathrm{ws}} v_{w}}}\right)}
$$

with $L_{\mathrm{ws}}=v_{w} /\left(\mathcal{R} \Gamma_{\mathrm{ws}}\right)$. Noting that $J_{\tau}<0$, we have to pick $s_{\tau}=-1$ for the sign of the CPV coupling, to get the right sign for the baryon asymmetry. The last factor in eq. (3.4) arises from

$$
I_{Y} \equiv \int \mathrm{d} z \mathrm{e}^{z\left(L_{S}^{-1}-\alpha_{-}\right)}=\frac{L_{\mathrm{ws}} L_{S}}{L_{S}+\frac{1}{2} L_{\mathrm{ws}}\left(1+\sqrt{1+\frac{4 D_{q}}{L_{\mathrm{ws}} v_{w}}}\right)} .
$$

\subsection{Comparison of approximations}

We have calculated the total baryon asymmetry using different approximations:

1. The analytical solution in eq. (3.4) of the purely leptonic transport equation, eq. (3.1), where the Higgs and quark sectors are neglected. We call this solution $A(l)$.

2. A numerical solution of eq. (3.1) called $N(l)$.

3. A numerical solutions that includes the Higgs and third-generation quarks called $N(l, h, q, t)$.

4. Finally, we investigate the different diffusion constants of left- and right-handed leptons. That is, we allow $D_{l} \neq D_{\tau}$ in $N(l, \tau)$. For numerical reasons we study this effect in the limit of a decoupled quark sector.

The entries in brackets in $A(\ldots)$ and $N(\ldots)$ denote the number densities for which we solve the transport equation explicitly in the respective approximation. In scenarios where the quark sector is considered, we apply local baryon conservation, eq. (2.16), and the relation in eq. (2.12) to account for the number densities of the bottom and lighter quarks.

The resulting baryon asymmetries in the various approximations for our benchmark point with $\Lambda_{\tau}=1 \mathrm{TeV}$ and bubble-wall velocity $v_{w}=0.05$ are given in table 1 . Comparing $\mathrm{N}(l, \tau)$ to $\mathrm{N}(l)$ shows that local lepton conservation is a reasonable approximation. More importantly, the analytical solution differs only $\sim 10 \%$ from the numerical solutions with 


\begin{tabular}{|l|c|c|c|c|}
\hline Approximation & $\mathrm{A}(l)$ & $\mathrm{N}(l)$ & $\mathrm{N}(l, h, q, t)$ & $\mathrm{N}(l, \tau)$ \\
\hline$Y_{B}$ & $6.8 \times 10^{-11}$ & $7.3 \times 10^{-11}$ & $7.3 \times 10^{-11}$ & $8.2 \times 10^{-11}$ \\
\hline
\end{tabular}

Table 1. Baryon asymmetry $Y_{B}$ for a tau CPV source obtained using various approximations as detailed in the main text. We used the benchmark values for the bubble-wall profile given in section 2.1 and $\Lambda_{\tau}=1 \mathrm{TeV}$.

\begin{tabular}{|l|l|r|}
\hline Length scale & Description & Benchmark value \\
\hline$L_{S}=\frac{2 L_{\mathrm{diff}}}{1+\sqrt{1+4 L_{\mathrm{diff}} / L_{\text {int }}^{S}}}$ & migration length in symmetric phase & 21.3 \\
\hline$L_{B}=\frac{2 L_{\mathrm{diff}}}{1+\sqrt{1+4 L_{\mathrm{diff}} / L_{\text {int }}^{B}}}$ & migration length in broken phase & 9.8 \\
\hline$L_{\mathrm{int}}^{S}=v_{w} / \bar{\Gamma}_{S}$ & interaction length in symmetric phase & 313.3 \\
\hline$L_{\mathrm{int}}^{B}=v_{w} / \bar{\Gamma}_{B}$ & interaction length in broken phase & 7.0 \\
\hline$L_{\mathrm{diff}}=D_{l} / v_{w}$ & lepton diffusion length & 22.7 \\
\hline$L_{\mathrm{diff}}^{q}=D_{q} / v_{w}$ & quark diffusion length & 1.4 \\
\hline$L_{\mathrm{ws}}=v_{w} /\left(\mathcal{R} \Gamma_{\mathrm{ws}}\right)$ & weak sphaleron length & 28.5 \\
\hline$L_{w}$ & bubble-wall width & 0.1 \\
\hline
\end{tabular}

Table 2. Length scales (in units of $\mathrm{GeV}^{-1}$ ) for a tau CPV source and their values for the benchmark values for the bubble-wall profile given in section 2.1 and $\Lambda_{\tau}=1 \mathrm{TeV}$.

and without inclusion of the Higgs and quark sectors, that is from $\mathrm{N}(l, h, q, t)$ and $\mathrm{N}(l)$ respectively. The tau Yukawa coupling is sufficiently small that decoupling the Higgs and quark sectors is an excellent approximation.

In the left panel of figure 1 the various approximations are compared while varying the value of the tau Yukawa coupling. The decoupling approximation works well up to $y_{\tau} \lesssim 0.2$ while for larger values significant Higgs and quark densities are generated. The right panel of figure 1 shows that the analytical approximation holds over a large range of bubble-wall velocities, such that the conclusions are not restricted to just our benchmark values.

\subsection{Parameter dependence}

Having found that the analytical solution provides an excellent approximation, we can use it to understand how the baryon asymmetry depends on various parameters, such as the Yukawa coupling and the parameters associated to the bubble wall. It is useful to rewrite the solution for $Y_{B}$ in eq. (3.4) in terms of various length scales

$$
Y_{B}=\frac{3}{s \mathcal{R}} \frac{\bar{S}}{v_{w} L_{\mathrm{ws}}} \frac{1}{\sqrt{1+4 \frac{L_{\mathrm{diff}}}{L_{\mathrm{int}}^{S}}}+\sqrt{1+4 \frac{L_{\mathrm{diff}}}{L_{\mathrm{int}}^{B}}}} \frac{L_{\mathrm{ws}} L_{S}}{L_{S}+\frac{1}{2} L_{\mathrm{ws}}\left(1+\sqrt{1+4 \frac{L_{\mathrm{diff}}^{q}}{L_{\mathrm{ws}}}}\right)},
$$

which are defined in table 2. $L_{S}$ and $L_{B}$ determine how far the asymmetry migrates into, respectively, the symmetric and broken phase. On larger length scales the asymmetry is exponentially suppressed by the $\mathrm{e}^{-|z| / L_{i}}$-factor in eq. (3.2) and (3.3). $L_{\text {diff }}=D_{l} / v_{w}$ is the 
diffusion length, which determines how far the asymmetry can diffuse into the symmetric phase before it is overtaken by the expanding bubble.

The interaction lengths in the symmetric and broken phase are denoted by $L_{\text {int }}^{i}=v_{w} / \bar{\Gamma}_{i}$ with $i=S, B$, respectively. If interactions are slow, $L_{\text {diff }} \ll L_{\text {int }}^{i}$, they can be neglected, and the migration scale is determined by diffusion $L_{i} \simeq L_{\text {diff. }}$. In the opposite limit of fast interactions, the symmetry is washed out over scales larger than $L_{i} \simeq \sqrt{L_{\mathrm{diff}} L_{\text {int }}^{i}}=$ $\sqrt{D_{l} / \bar{\Gamma}_{i}}$. In the broken phase, $L_{B}$ has to be compared with the spatial extend of the source, which is set by the bubble-wall width $L_{w}$. For $L_{w} \ll L_{B}$ the source is not diluted by diffusion nor interactions and we can approximate $\bar{S}$ by the first term in eq. (3.3).

The conversion of the chiral number into a net baryon number through eq. (2.19) introduces additional length scales: the baryon/quark diffusion length scale $L_{\text {diff }}^{q}=D_{q} / v_{w}$, and the scale on which the weak sphalerons act $L_{\mathrm{ws}}=v_{w} /\left(\mathcal{R} \Gamma_{\mathrm{ws}}\right)$. In the limit $L_{S} \ll$ $L_{\mathrm{ws}}$ the migration length, i.e., how far the chiral asymmetry migrates into the symmetric phase, determines the extend of the region in front of the bubble wall that contributes to the baryon asymmetry and the integral eq. (3.5) becomes $I_{Y} \simeq L_{S}$. In the opposite limit, $L_{\mathrm{ws}} \ll L_{S}$, it seems necessary to include the weak sphaleron interactions directly in the transport equations, as there is no hierarchy between the sphaleron and Yukawa interactions, invalidating the two step procedure. However, although both processes may be important for the final asymmetry (depending on the bubble-wall velocity) they are still very far from equilibrium, and back reaction effects are small. We checked numerically that the two-step procedure used to derive eq. (3.4) is a good approximation to the full coupled equations, see section $\mathrm{B}$ for more details. We then find that in this regime the integral is cut off by $L_{\mathrm{ws}}$, the scale on which the weak sphalerons act, and $I_{Y} \simeq L_{\mathrm{ws}}(1+\sqrt{r})^{-1}$ in the parameter regime of interest, with

$$
r=\frac{L_{\mathrm{ws}} L_{\mathrm{diff}}^{q}}{L_{S}^{2}} \stackrel{v_{w \rightarrow 0}}{=} \frac{D_{q}}{D_{l}} \frac{\bar{\Gamma}_{S}}{\mathcal{R} \Gamma_{\mathrm{ws}}},
$$

where the right-hand expression is valid in the small-velocity limit where $r$ is maximal. For the tau Yukawa interactions $r \ll 1$ and we can neglect the baryon diffusion effects, and the approximation made in eq. (2.19) holds. This is no longer the case for the topand bottom-source scenarios discussed in section 4, as can be anticipated from the above estimate. Replacing the tau Yukawa interaction with the top Yukawa or strong sphaleron interaction, and setting $D_{l} \rightarrow D_{q}$ gives $r \gg 1$.

The various length scales are listed in table 2 , along with their value for the benchmark point. The length scales depend on bubble-wall parameters such as $v_{N}$ and $v_{w}$ that we can vary depending on the electroweak phase transition, and on SM parameters such as the tau Yukawa coupling, $y_{\tau}$. We also consider variations in $y_{\tau}$ to see what would happen for source terms involving lighter SM fermions and to get some (limited) insight about what happens for heavier quarks, as further discussed in the next section. For all parameter choices, we have $L_{\text {int }}^{B} \ll L_{\text {int }}^{S}$ as $\Gamma_{M}^{(l)}$ greatly exceeds the Yukawa interaction rate in both the symmetric and broken phase. The solution for the baryon asymmetry can then be 
divided into three ${ }^{2}$ different regions, depending on the chosen parameters and the resulting sizes of the relevant length scales:

a $\quad L_{\text {diff }}<L_{\text {int }}^{B}, L_{\text {int }}^{S}, L_{\mathrm{ws}}$ which corresponds to small Yukawa couplings and a large bubble-wall velocity. Interactions are slow and can be neglected, and the chiral asymmetry diffuses without washout $L_{i} \simeq L_{\text {diff. Since the diffusion length is much }}$ larger than the source width $L_{\text {diff }} \gg L_{w}$ there is no dilution of the source and the expansion in eq. (3.3) is valid. In this regime, the baryon asymmetry becomes

$$
Y_{B} \simeq \frac{3}{2 s \mathcal{R}} \frac{\bar{S}}{v_{w}} \frac{L_{\mathrm{diff}}}{L_{\mathrm{ws}}} \propto \frac{y_{\tau}^{2}}{v_{w}^{2}} \frac{1}{\Lambda_{\tau}^{2}}
$$

The asymmetry decreases for large bubble-wall velocity, which can be understood as the faster the bubble moves, the less time there is for the chiral asymmetry to diffuse into the symmetric phase and to be converted into a baryon asymmetry. The asymmetry decreases quadratically with a smaller Yukawa coupling, which originates from the scaling of the source. In practice, the tau Yukawa coupling is slightly too large for the above scaling to hold for the benchmark velocity $v_{w} \simeq 0.05$.

b $\quad L_{\text {int }}^{B}<L_{\text {diff }}<L_{\text {int }}^{S}, L_{\text {ws }}$. Interactions are now important in the broken phase and $L_{B} \simeq \sqrt{L_{\mathrm{int}}^{B} L_{\mathrm{diff}}}$. The dilution of the source in $\bar{S}$ is still a negligible effect, as $L_{B} \simeq L_{w}$ only holds for $\mathcal{O}(1)$ Yukawa couplings. The interactions in the broken phase, however, change the baryon asymmetry into

$$
Y_{B} \simeq \frac{3}{2 s \mathcal{R}} \frac{\bar{S}}{v_{w}} \frac{\sqrt{L_{\mathrm{diff}} L_{\mathrm{int}}^{B}}}{L_{\mathrm{ws}}} \propto \frac{y_{\tau}}{v_{w}} \frac{1}{\Lambda_{\tau}^{2}} .
$$

$Y_{B}$ thus scales linearly with the Yukawa coupling and inversely with the bubble velocity. Here we see for the first time a violation of the naive $y_{f}^{2}$ scaling of the asymmetry.

c $\quad L_{\text {int }}^{B}<L_{\text {ws }}<L_{\text {diff, }}, L_{\text {int }}^{S}$ which corresponds to small velocities (and $y_{\tau} \lesssim 0.2$ such that the analytical approximation is valid). The baryon asymmetry

$$
Y_{B} \simeq \frac{3}{2 s \mathcal{R}} \frac{\bar{S}}{v_{w}} \sqrt{\frac{L_{\mathrm{int}}^{B}}{L_{\mathrm{diff}}}} \propto \frac{y_{\tau} v_{w}}{\Lambda_{\tau}^{2}},
$$

now scales linearly with both $y_{\tau}$ and $v_{w}$. The asymmetry decreases for small velocity, as in this limit the evolution approaches thermal equilibrium. We neglect the $L_{\text {int }}^{S}$ dependence in the denominator in eq. (3.4) as it is subdominant to the $L_{\mathrm{int}}^{B}$ term.

Comparing eqs. (3.9) and (3.10) it follows that the asymmetry is maximized as a function of the velocity at the boundary of the two regimes, that is, for $L_{\mathrm{ws}} \sim L_{\text {diff. For }}$

\footnotetext{
${ }^{2}$ In principle there exists a fourth region $L_{\text {int }}^{S} \ll L_{\text {diff }}$ and $L_{S}=\sqrt{L_{\text {int }}^{S} L_{\text {diff }}} \ll L_{\text {ws }}$. This requires $\mathcal{O}(1)$ Yukawa couplings, for which our analytical approximation eq. (3.2) breaks down. As this is not the physical region of interest, we do not discuss this possibility further.
} 

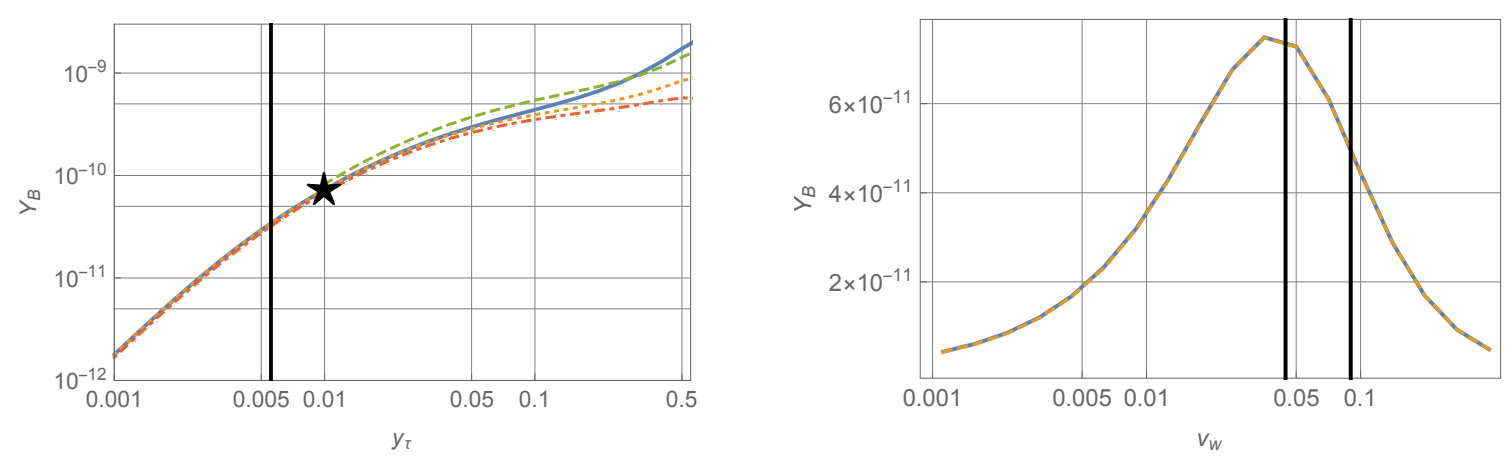

Figure 1. Left: $Y_{B}$ as a function of the Yukawa coupling $y_{\tau}$ for the same four approximations as in table 1 (solid blue: $\mathrm{N}(l, h, q, t)$, dotted orange: $\mathrm{N}(l)$, dashed green $\mathrm{N}(l, \tau)$, dotdashed red: $\mathrm{A}(l))$. The region left (right) of the vertical black line corresponds to region $\mathbf{a}(\mathbf{b})$. The star denotes the SM value of $y_{\tau}$. Right: $Y_{B}$ as a function of the bubble-wall velocity $v_{w}$ (solid blue: $N(l, h, q, t)$, dashed orange: $\mathrm{N}(l))$. The left, middle and right regions correspond to regions $\mathbf{c}, \mathbf{b}$, and $\mathbf{a}$, respectively.

the SM tau Yukawa coupling, region a corresponds to $v_{w} \gtrsim 0.1$, region $\mathbf{b}$ to $0.04 \lesssim v_{w} \lesssim 0.1$, and region $\mathbf{c}$ to $v_{w} \lesssim 0.04$.

The scaling of the asymmetry with bubble-wall velocity and Yukawa coupling are illustrated in figure 1. The left panel shows $Y_{B}$ as a function of the Yukawa coupling $y_{\tau}$. The vertical thick line gives the boundary between region $\mathbf{a}$ and $\mathbf{b}$; region $\mathbf{c}$ does not occur for our benchmark velocity. In region a, on the left of the vertical thick line, the baryon asymmetry scales quadratically with the small Yukawa coupling, while in region $\mathbf{b}$, on the right, the scaling is linear. The SM Yukawa value $y_{\tau}=0.01$ falls in region $\mathbf{b}$, and the baryon asymmetry for this value is indicated by a star (and corresponds to our benchmark number in table 1). The most relevant observation is that for $y_{\tau} \geq 0.005, Y_{B}$ only grows linearly with $y_{\tau}$ instead of the naively expected quadratic scaling. This confirms that CPV sources for fermions lighter than top quarks are less disadvantageous than might be expected.

The right panel shows $Y_{B}$ as a function of the bubble-wall velocity $v_{w}$. Now the black lines divide, from right to left, regions $\mathbf{a}, \mathbf{b}$, and $\mathbf{c}$ that scale respectively as $v_{w}^{-2}, v_{w}^{-1}$ and $v_{w}$. The boundary between $\mathbf{b}$ an $\mathbf{c}$ corresponds to the velocities for which $L_{\mathrm{ws}} \sim L_{\text {diff }}$, close to which the baryon asymmetry is maximized. Our benchmark point lies in scenario b, very close to the optimum value.

We end this section with a short discussion of how the baryon asymmetry depends on the dynamics of the first-order phase transition that we have parameterized by the bubblewall profile eq. (2.1). The bubble-wall width only enters the equations via the source. In the limit that $L_{w} / L_{B} \ll 1$, as appropriate for a tau source, the dependence on this parameter cancels. A larger bubble width gives a wider source but with a smaller amplitude, such that the total integrated source $\bar{S}$ remains the same.

The source scales as $\bar{S} \propto v_{N}^{4}$ with $v_{N}$ the Higgs vev during nucleation; this power comes about because of the structure of our dimension-six CPV operator which involves three Higgs fields. ${ }^{3}$ The relaxation rate $\Gamma_{M}^{(f)} \propto v_{N}^{2}$ depends on the vev as well. It thus follows

\footnotetext{
${ }^{3}$ In two-Higgs doublet models CPV can originate from dim-4 operators, in which case the corresponding source would only scale quadratically with the vev.
} 
that in region $\mathbf{a}$, the baryon asymmetry scales as $Y_{B} \propto v_{N}^{4}$ and in region $\mathbf{b}$ as $Y_{B} \propto v_{N}^{3}$. For the SM tau Yukawa coupling we are in region $\mathbf{b}$, but the velocity dependence of $L_{B}$ cannot be fully neglected and numerically we find a scaling $Y_{B} \propto v_{N}^{3.2}$. Although the dependence on the vev is strong, we do not expect $v_{N}$ to vary too much between different BSM models.

The dependence on the nucleation temperature $T_{N}$ is harder to estimate, as many parameters, such as the thermal masses and thermal width, depend on the temperature. All interaction rates scale $\Gamma_{i} \propto T$, except for $\Gamma_{M} \propto 1 / T$; further $D_{i} \propto 1 / T$ and $S \propto T$. With this scaling we find that $Y_{B} \propto 1 / T_{N}^{2}$ in regime a and $Y_{B} \propto 1 / T_{N}$ in regime b. We note that as $T_{N}$ increases the relative importance of $L_{\text {int }}^{B}$ decreases, and we move from regime $\mathbf{b}$ to $\mathbf{a}$.

\subsection{Producing the universal baryon asymmetry with a tau source}

Can CP violation in the lepton sector produce the observed baryon asymmetry? The asymmetry for the benchmark point in table 1 is fairly close to the observed value in eq. (1.1). The scale $\Lambda_{\tau} \sim 1 \mathrm{TeV}$ if fairly low, but not inconsistent with EDM and collider experiments. It could be even lowered somewhat to further increase $Y_{B}$ but the EFT description becomes problematic for such low scales. As shown in the right panel of figure 1 the benchmark $v_{w}=0.05$ is already close to optimal, and there is little room for improvement. Definite statements about the viability of the scenario are not easy to make, as the performed calculations still suffer from uncertainties, such as those related to the description of the phase transition, that are hard to quantify. We refer to e.g. ref. [19] for a more general discussion of outstanding problems in calculations of EWBG. Nevertheless, our analysis indicates that CPV sources in the tau sector are viable despite the small Yukawa coupling and not yet significantly constrained by EDM experiments.

Our study of the dependence of $Y_{B}$ on the value of $y_{\tau}$ can be directly used to study the cases of $\mathrm{CP}$-violating electron and muon source terms. In such cases, the transport equations are identical after replacing the third-generation lepton number densities with the first- or second-generation densities and rescaling the $y_{\tau} \rightarrow y_{\mu, e}$. The left panel of figure 1 shows that for Yukawa couplings $y_{l}$ larger than $y_{\tau}$ we are in regime $\mathbf{b}$ where $Y_{B}$ grows linear with $y_{l}$. For smaller Yukawa couplings however, we are in regime a, where $Y_{B}$ decreases as $y_{l}^{2}$. For equal values of the scale of new physics $\Lambda_{e}=\Lambda_{\mu}=\Lambda_{\tau}$ the values of $Y_{B}$ in the electron and muon scenarios are then suppressed by a factor $\left(y_{e} / y_{\tau}\right)^{2} \simeq 8 \cdot 10^{-8}$ and $\left(y_{\mu} / y_{\tau}\right)^{2} \simeq 4 \cdot 10^{-3}$, respectively. For the electron, EDM constraints require $\Lambda_{e}>5.7 \mathrm{TeV}$, which suppresses the baryon asymmetry even more. The resulting values of $Y_{B}$ are far below the observed asymmetries and we conclude that electron and muon CPV sources do not lead to successful baryogenesis.

\section{Baryogenesis with a quark source}

In this section we discuss baryogenesis with a quark CPV source and at first neglect the additional lepton-quark interaction in section 2.7. We start the discussion with a CPV source in the top-quark sector. This scenario has been discussed extensively in the literature, see for example refs. $[15,79,80]$ where the same dimension-six CPV operator has 


\begin{tabular}{|l|c|c|c|c|c|}
\hline Approximation & $\mathrm{FR}(q)$ & $\mathrm{N}(q, t, h)$ & $\mathrm{N}(q, t, h, u)$ & $\mathrm{N}(q, t, h, u, l)$ & $\mathrm{A}(q, t, h, u, l)$ \\
\hline$Y_{B}$ & $1.6 \times 10^{-12}$ & $3.5 \times 10^{-13}$ & $3.4 \times 10^{-13}$ & $1.5 \times 10^{-12}$ & $1.1 \times 10^{-12}$ \\
\hline
\end{tabular}

Table 3. Baryon asymmetry $Y_{B}$ for a top source using different approximations discussed in the main text. We used the benchmark values for the bubble-wall profile given in section 2.1 and $\Lambda_{t}=7.1 \mathrm{TeV}$ consistent with EDM experiments.

been considered. Since the source is proportional to the value of the Yukawa coupling squared $S_{f} \propto y_{f}^{2}$, the baryon asymmetry generated by a top source can be expected to be larger than for the corresponding source terms involving lighter fermions. But several factors that were identified in section 2.6 suppress the corresponding BAU. We therefore study how effective a top source actually is. In the process we investigate the role of SM bottom and tau Yukawa interactions that are typically neglected.

A CPV bottom source is suppressed by $\left(y_{b} / y_{t}\right)^{2} \simeq 4 \times 10^{-4}$ with respect to a top source. Part of this suppression can be compensated as EDM experiments do not set as stringent constraints on the CPV bottom source. In addition, a smaller Yukawa coupling also implies less washout because $\Gamma_{M}^{(b)}$ is smaller and, as demonstrated for a tau source, it is not immediately clear how $Y_{B}$ varies with the size of the Yukawa coupling, and thus how the top and bottom scenarios compare. While the bottom Yukawa coupling is still roughly a factor two larger than the tau Yukawa coupling, we nevertheless expect the asymmetry to be suppressed with respect to the tau source, because the bottom has strong sphaleron interactions and a smaller diffusion constant leading to less efficient generation of $Y_{B}$.

\subsection{Top source}

We calculate the baryon asymmetry arising from a top CPV source using various approximations:

1. The simplest approximation is to first neglect the small bottom and tau Yukawa couplings. Using eq. (2.12) and (2.16) leaves us with three transport equations for the number densities $q, t$, and $h$. Finally, we apply the often-used fast-rate approximation which assumes that the top Yukawa and strong sphaleron transitions are fast and (nearly) in equilibrium [60, 77]. This leaves us with a single equation for $q$ that can be solved analytically. We denote the solution by $\operatorname{FR}(q)$.

2. We neglect bottom and tau Yukawa couplings, but do not use the fast-rate approximation. Instead we numerically solve the set of three transport equations for $q, t$, and $h$ and call the solution $\mathrm{N}(q, t, h)$.

3. Next we include the effects of the bottom Yukawa. We can no longer connect the $b$ density to those of light quarks and the transport equation for $u$ has to be included. The numerical solution of the four transport equations is called $\mathrm{N}(q, t, h, u)$.

4. We also include the tau Yukawa coupling and add the $l$ transport equation. In principle, we should keep the right-handed lepton fields too, but, as discussed in the 
tau scenario, the approximation of local lepton number conservation, $D_{l}=D_{\tau}$, is reasonable and we can eliminate the right-handed leptons. The solution is called $\mathrm{N}(q, t, h, u, l)$.

5. We solve the same set of equations using the semi-analytical method [73], which neglects the variation of the rates over the bubble wall, and write this solution as $\mathrm{A}(q, t, h, u, l)$.

As before between brackets in $\mathrm{A}(\ldots)$ and $\mathrm{N}(\ldots)$ we list the transport equations that we solve explicitly. The obtained values for $Y_{B}$ for our benchmark point with $\Lambda_{t}=7.1 \times$ $10^{3} \mathrm{GeV}$ and bubble-wall velocity $v_{w}=0.05$ in the various approximations are listed in table 3. To get the right sign for $Y_{B}$, the phase in the CPV operator in eq. (2.2) is set to $s_{t}=1$.

The fast-rate approximation assumes thermal equilibrium for the strong sphaleron and top Yukawa rates, but this approximation is invalid close to the bubble wall in the symmetric phase and invalid everywhere in the broken phase where $\Gamma_{M}^{(t)}$ is instead the fastest rate. By comparing the solutions $\operatorname{FR}(q)$ and $\mathrm{N}(q, t, h)$ we see that the fast-rate approximation overestimates $Y_{B}$ by roughly a factor five and is thus a poor approximation, in agreement with the findings of ref. [73]. The fast-rate approximation should be avoided to calculate $Y_{B}$, especially since it does not represent a systematic expansion and as such the associated error cannot be systematically estimated.

By comparing $\mathrm{N}(q, t, h)$ and $\mathrm{N}(q, t, h, u)$ we see that including bottom Yukawa interactions only provides a few-percent correction. Considering the significant intrinsic uncertainties associated to EWBG calculations, neglecting the bottom Yukawa seems a good approximation. It might come as a surprise then that including the even smaller tau Yukawa interaction has a much greater impact. The baryon asymmetry associated to $\mathrm{N}(q, t, h, u, l)$ is roughly five times that of $\mathrm{N}(q, t, h, u)$. Where does this lepton-induced enhancement stem from?

As discussed in section 2.6, when the strong sphaleron interactions are (approximately) in thermal equilibrium, $\mu_{\mathrm{ss}} \simeq 0$, the total chiral asymmetry in quarks is highly suppressed. This is illustrated in the left panel of figure 2. The red line depicts the chiral asymmetry in just top quarks, $q(z)$, as a function of the distance to the bubble wall in the symmetric phase. This individual asymmetry is everywhere much larger than the total chiral asymmetry in the quark sector, $n_{L}^{(q)}(z) \equiv \sum_{i} q_{i}$, where the sum runs over the three generations. $n_{L}^{(q)}(z)$ is plotted in orange and is itself larger than the chiral asymmetry in tau leptons, $l(z)$, depicted in green, up to $|z| \simeq 5$. For larger distance $l(z)$ becomes larger and consequently dominates the total chiral asymmetry, $n_{L}(z)=n_{L}^{(q)}(z)+l(z)$, depicted in blue.

The distance from the bubble in the symmetric phase at which point the various densities start to drop can be understood qualitatively from the analytical solution for the lepton source discussed in the previous section. In analogy with the leptonic case there are three important length scales: the diffusion length $L_{\text {diff }}^{i} \sim D_{i} / v_{w}$, the migration length set by strong sphaleron interactions $L_{S}^{(\mathrm{SS})} \sim \sqrt{k_{q} D_{q} / \Gamma_{\mathrm{ss}}}=\mathcal{O}(1) / \mathrm{GeV}$, and the migration length set by the top Yukawa interactions $L_{S}^{\left(y_{t}\right)} \sim \sqrt{k_{q} D_{q} / \Gamma_{y}^{(t)}}=\mathcal{O}(0.1) / \mathrm{GeV}$. The Yukawa interactions are strongest and provide the dominant source of washout near 

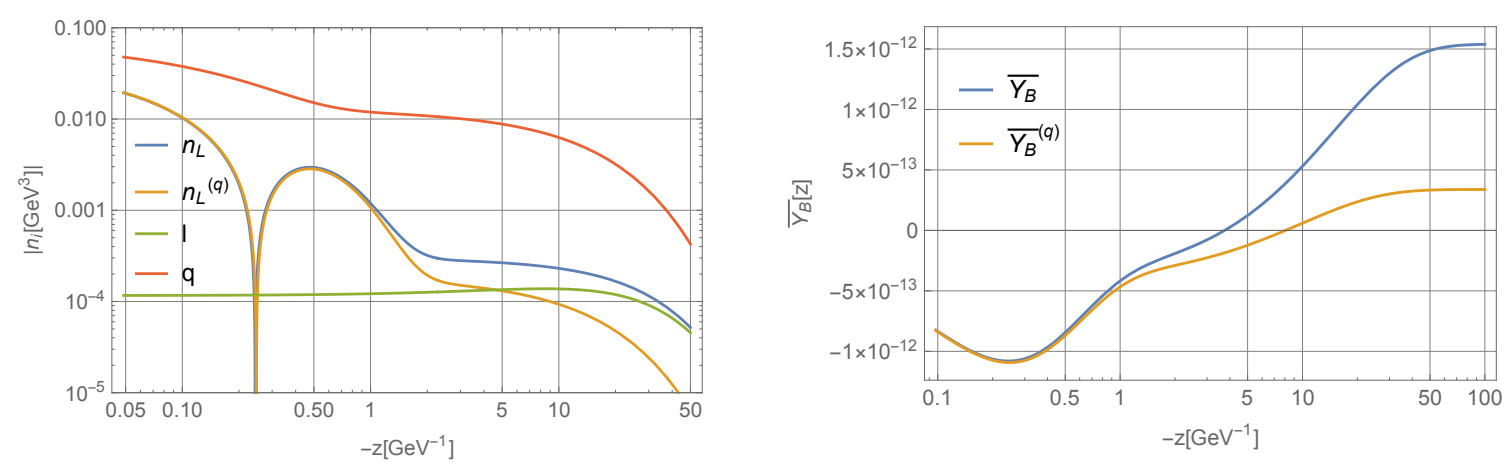

Figure 2. Left plot: absolute value of the number densities $n_{L}, n_{L}^{(q)}, l$, and $q$ in the symmetric phase for a top source. The computation includes top, bottom and tau Yukawa interactions. Right plot: baryon asymmetry $\bar{Y}_{B}(z)$ and $\bar{Y}_{B}^{(q)}(z)$ as a function of the integration cut-off.

the bubble wall. This washout leads to the decrease of $n_{L}$ for small values of $z$ and the associated zero-crossing around $|z|=0.2 / \mathrm{GeV}$. At distances around $|z| \sim L_{S}^{\left(y_{t}\right)}$, the top Yukawa interactions are in equilibrium and the $n_{L}$ would plateau to a constant value. However, this is not clear in figure 2 where $n_{L}$ keeps decreasing because of washout from strong sphalerons which is active up to the larger distance $L_{S}^{(\mathrm{SS})}$. This washout, and the related decrease of $n_{L}$, ends for $|z|>L_{S}^{(\mathrm{SS})}$.

The individual particle densities migrate much further in the symmetric phase than the total quark chiral asymmetry, as their spread is only limited by diffusion. Indeed with both the top Yukawa and strong sphaleron interactions in equilibrium, the number densities of the left- and right-handed quarks and the Higgs are related (by the condition that the chemical potential vanishes). Physically what happens is that on the relevant time scales, quarks and Higgses are converted into each other instantly, and they behave as a single degree of freedom that diffuses into the symmetric phase. Their diffusion length, which determines how far the densities extend, is dominated by the Higgs diffusion length which is large as the Higgs does not feel strong interactions. As such $L_{\text {diff }} \sim D_{h} / v_{w} \simeq 20 / \mathrm{GeV}$. A more precise estimate is obtained from the transport equation for the single degree of freedom, which gives (see eq. (77a) in [60]) $L_{\text {diff }} \simeq 16 / \mathrm{GeV}$.

Finally, the tau Yukawa interactions are out of equilibrium throughout. The lepton number density slightly increases away from the bubble wall as there is more time to convert Higgs quanta into leptons. The lepton asymmetry migrates over distances set by the lepton diffusion length $L_{\text {diff }} \sim D_{l} / v_{w} \simeq 20 / \mathrm{GeV}$. Because of the small tau Yukawa coupling the lepton density is always much smaller than the left-handed top density $q$. However, it is the chiral density that matters, and given the very efficient suppression of the chiral asymmetry in quarks, leptons actually start to dominate $n_{L}$ for $|z| \gtrsim L_{S}^{(\mathrm{SS})}$. This explains why the inclusion of leptons can give a sizeable correction to the final baryon asymmetry.

What is maybe surprising is that the leptons actually give the dominant contribution for our benchmark point. The reason for this is that $n_{L}^{(q)}$ crosses zero close to the bubble wall around $|z|=0.25 / \mathrm{GeV}$. In the right panel of figure 2 we show the contribution to the 


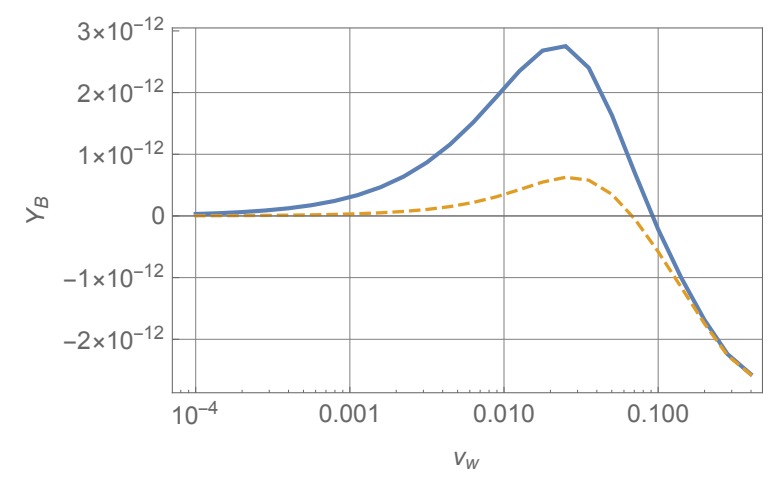

Figure 3. $Y_{B}$ as a function of velocity $v_{w}$ with (solid blue) and without (dashed orange) lepton interaction included.

asymmetry from the wall up to a distance $z$. That is, we plot the function

$$
\bar{Y}_{B}(z)=-\frac{3 \Gamma_{\mathrm{ws}}}{2 s D_{q} \alpha_{+}} \int_{z}^{0} \mathrm{~d} z^{\prime} n_{L}\left(z^{\prime}\right) \mathrm{e}^{-\alpha_{-} z^{\prime}},
$$

with $\alpha_{ \pm}$given in eq. (2.18). For our benchmark parameters, the chiral asymmetry close to the bubble wall $n_{L} \simeq n_{L}^{(q)}$ gives a negative contribution to the integral in eq. (2.19). However, past the zero-crossing the contribution becomes positive. Due to the sign change, the total asymmetry generated up to $z=10 / \mathrm{GeV}$ from just $n_{L}^{(q)}$ vanishes. Around this point, the chiral asymmetry in quarks is small and leptons dominate the chiral asymmetry. Integrating over larger distances leptons then give the dominant contribution to $Y_{B}$. This sensitivity of $Y_{B}$ to the tau Yukawa interactions is thus twofold: 1) even though small, they are the only mechanism via which the chiral asymmetry is transferred from the quark sector, where the CPV source is located, to the lepton sector, where they escape the efficient washout by strong sphalerons. 2) There is a cancellation of the contribution of $n_{L}^{(q)}$ to the baryon asymmetry.

We can now also understand why including bottom Yukawa interactions has less impact. These will transfer some of the top chiral asymmetry into a bottom chiral asymmetry, but the total is still washed out by strong sphaleron interactions. This transfer therefore does not lead to a significant change in $Y_{B}$.

An interesting question is now whether including tau leptons is generally important or whether it is a special feature of our benchmark parameters. To study this, we calculate $Y_{B}$ associated to the $\mathrm{N}(q, t, h, u)$ and $\mathrm{N}(q, t, h, u, l)$ solutions for a wide range of bubblewall velocities. The values of $Y_{B}$ are plotted in figure 3 . We conclude that the lepton contribution is important for a large range of velocities $v_{w} \sim 10^{-4}-10^{-1}$, but becomes less relevant for larger velocities. For such large velocities the baryon asymmetry is dominated by the chiral asymmetry close to the bubble wall, where the leptons contribute little. In this case, the integral is cut off by the diffusion length $L_{\text {diff }} \propto 1 / v_{w}$, which becomes small.

\subsection{Producing the universal baryon asymmetry with a top source}

Let us finally answer whether a top source can produce the observed baryon asymmetry in eq. (1.1). For equal Wilson coefficients of the dimension-six CPV operators, $\Lambda_{t}=\Lambda_{\tau}$, the 


\begin{tabular}{|l|c|c|c|}
\hline Approximation & $\mathrm{N}(q, t, h, b)$ & $\mathrm{N}(q, t, h, b, l)$ & $\mathrm{A}(q, t, h, b)$ \\
\hline$Y_{B}$ & $8.3 \times 10^{-13}$ & $8.4 \times 10^{-13}$ & $7.3 \times 10^{-13}$ \\
\hline
\end{tabular}

Table 4. Baryon asymmetry $Y_{B}$ for a bottom source with $\Lambda_{b}=1 \mathrm{TeV}$ and $v_{w}=0.05$ using different approximations discussed in the text.

asymmetry induced by the top source is of the same order as for the tau source. However, for taus we can choose a fairly low scale $\Lambda_{\tau} \sim 1 \mathrm{TeV}$, while EDM experiments constrain $\Lambda_{t} \geq 7.1 \mathrm{TeV}$. As such, the asymmetry in our benchmark scenario is almost two orders of magnitude too small. As the asymmetry is rather sensitive to the Higgs vev at nucleation, this might be the easiest parameter to adjust to boost the asymmetry, but how much this is allowed depends on the details of the scalar sector that we have not specified. Our chosen bubble-wall velocity is already close to the optimal value as shown in figure 3. All things considered it seems unlikely that the dimension-six top Yukawa interaction can still lead to sufficient asymmetry.

It must be said, that the uncertainties in the calculation for a top CPV source are large. The vev-insertion approximation is dubious as the top Yukawa coupling is not small. Another potentially large effect can be the inclusion of collective plasma effects, the so-called hole modes, in the calculation of the source term and interaction rates, see the discussion in ref. [19]. Despite these caveats, it is fair to say that the observed baryon asymmetry is obtained more easily using a tau source, mainly due to the significant EDM constraints. In section 5 we discuss ways of boosting the baryon asymmetry for top-source scenarios by adding additional BSM lepton-quark interactions. Other ways out could be by considering CP-violating interactions between top quarks and new scalar fields as such interactions are not directly constrained by EDMs if the new scalar field does not couple to electrons. Such a setup can appear in, for example, two-Higgs doublet models although several couplings have to be set to very small values by hand.

\subsection{Bottom source}

We now turn to the bottom source and investigate whether such a source can be as efficient as a tau source. To calculate the chiral asymmetry generated by a bottom source, one can use the same set of transport equations as for the top source. We calculate the asymmetry first by neglecting leptons and write this solution as $\mathrm{N}(q, t, h, u)$. We then include tau Yukawa interactions and add the $l$-equation to the set of transport equations and obtain the solution $\mathrm{N}(q, t, h, u, l)$. The corresponding semi-analytical solution is called $\mathrm{A}(q, t, h, u, l)$. The baryon asymmetry for our benchmark point with $\Lambda_{b}=1 \mathrm{TeV}$ and bubble-wall velocity $v_{w}=0.05$ is listed in table 4 (with $s_{b}=-1$ the sign of the dimension-six operator).

From the table it is clear that the asymmetry from a bottom source is almost two orders of magnitude smaller than the baryon asymmetry from a tau source for the same scale $\Lambda_{b}=\Lambda_{\tau}$. Just as for the top source, the chiral asymmetry in bottom quarks is effectively erased by the strong sphaleron interactions, which become important at $|z| \sim$ $L_{S}^{(\mathrm{SS})}$. Beyond this scale the leptons again give the dominant contribution to the chiral asymmetry, as can be seen in the left panel in figure 4. However, unlike the top-source 

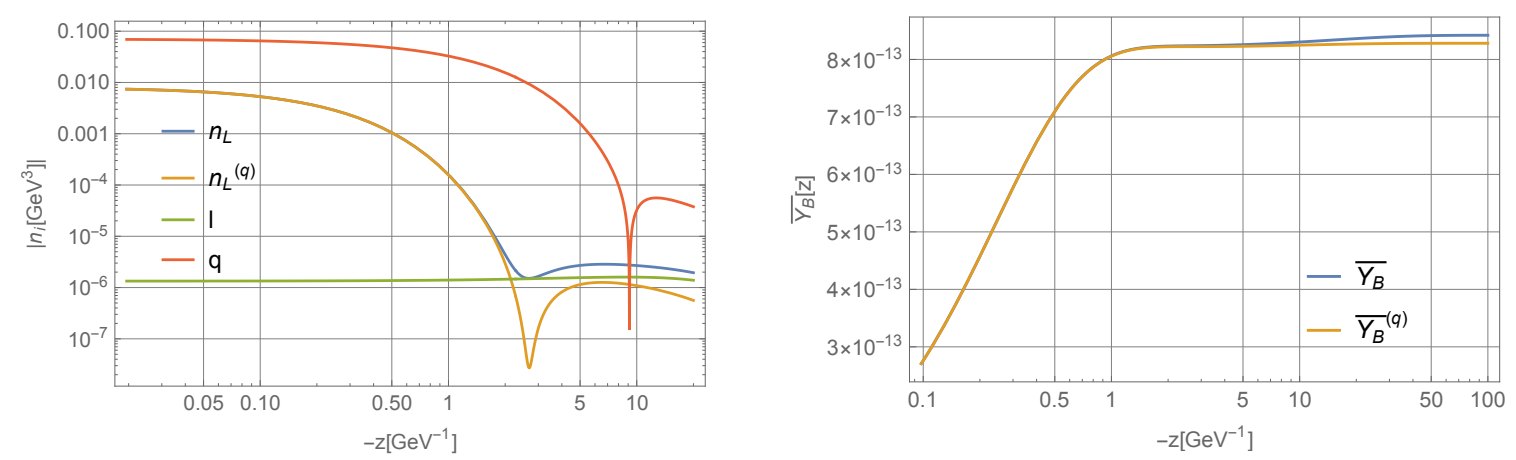

Figure 4. Left plot: absolute value of the number densities $n_{L}, n_{L}^{(q)}, l$ and $q$ in the symmetric phase for a bottom source. The computation includes top, bottom and tau Yukawa interactions. Right plot: baryon asymmetry $\bar{Y}_{B}(z)$ and $\bar{Y}_{B}^{(q)}$ as a function of the integration cut-off.

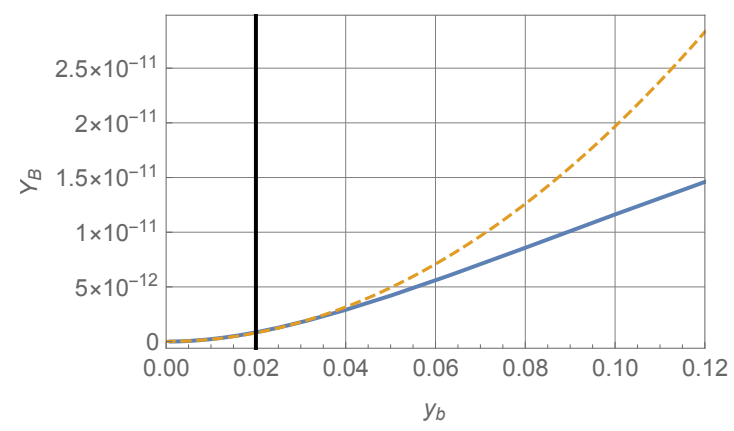

Figure 5. Baryon asymmetry for a bottom source as a function of the bottom Yukawa coupling $y_{b}$ (solid blue line). The orange dashed line shows a quadratic fit and the black vertical line indicates the SM value of $y_{b}$ at the electroweak scale.

case, there is no zero-crossing, and thus no cancellation in $Y_{B}$. Now, the total baryon asymmetry is dominated by the contribution close to the bubble wall, where leptons are negligible, see the right panel in figure 4.

We conclude that the bottom source produces a value of $Y_{B}$ that is about two orders of magnitude too small. What about sources involving even lighter quarks? In figure 5, the solid blue line depicts the value of $Y_{B}$ as a function of the bottom Yukawa coupling $y_{b}$ at the electroweak scale. The orange dashed line is a fit to a quadratic function of $y_{b}$. Effectively, for the SM value of $y_{b}$ and smaller values we are in regime a discussed in section 3.3 where $Y_{B} \propto y_{b}^{2}$. Since the system of transport equations for an up, down, strange, or charm source is very similar to that of a bottom source, we expect that sources involving light quarks will be suppressed by $\left(y_{q} / y_{b}\right)^{2}$ compared to the numbers in table 4 . Such sources will thus provide negligible contributions to $Y_{B}$. For $y_{b} \geq 0.04$ the asymmetry starts to scale linearly $Y_{B} \propto y_{b}$ (the system enters regime $\mathbf{b}$ discussed in section 3.3). The linear instead of quadratic scaling partially explains why a top source is not as effective compared to a bottom source as might be expected from just looking at the CPV source term. 


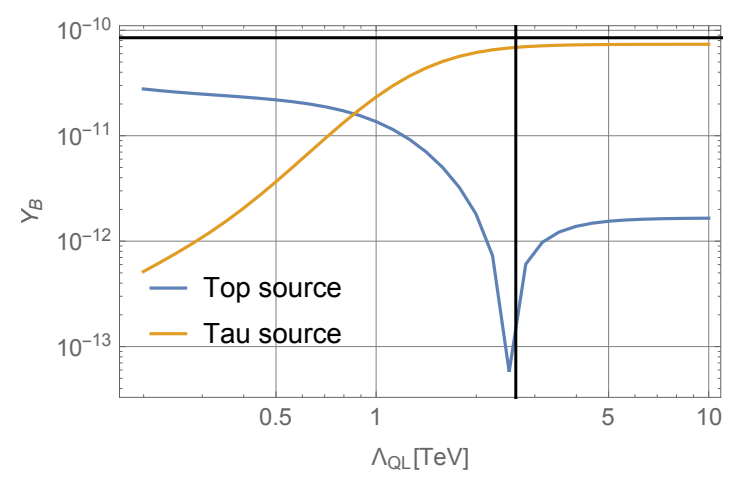

Figure 6. $Y_{B}$ as function of $\Lambda_{\mathrm{QL}}$ for a top source with $\Lambda_{t}=7.1 \times 10^{3} \mathrm{GeV}$ (blue) and a lepton source with $\Lambda_{\tau}=1 \times 10^{3} \mathrm{GeV}$ (yellow). The vertical black line corresponds to the new interactions rate being equal to the lepton Yukawa interaction rate. The horizontal black line corresponds to the measured value of the baryon asymmetry.

\section{Consequences of additional quark-lepton interactions}

In the previous sections we have argued that CP-violating leptonic sources are more effective than quark sources in generating a net baryon asymmetry. In this section, we analyze how we can enhance the asymmetry by considering BSM interactions that transfer the chiral asymmetry from quarks into leptons (and vice versa). Such interactions can be induced in BSM models by the exchange of new particles such as additional scalar bosons or leptoquarks. In particular, we consider the effect of turning on a new top-tau coupling, which we parameterize by the dimension-six operator in eq. (2.23).

It is intuitively clear what to expect. For a top source, the chiral asymmetry in quarks is effectively washed out by the top Yukawa and especially by the strong sphaleron interactions. In such a scenario, any part of the chiral asymmetry that is transferred to the lepton sector survives this washout, and the more effectively this is done the larger the final baryon asymmetry. We thus expect an increase in $Y_{B}$ for scales $\Lambda \lesssim 3 \mathrm{TeV}$ for which the new interaction rate becomes larger than the tau Yukawa interaction rate. For the lepton-source scenario, we expect instead a decrease in the baryon asymmetry. The new interaction, if large, will transfer part of the chiral asymmetry from the lepton to the quark sector, where it is effectively washed out. This behavior is indeed borne out by our numerical simulations shown in figure 6 , which shows baryon asymmetry for a lepton (yellow line) and top-quark (blue line) source, for $\Lambda_{\tau}=1 \mathrm{TeV}$ and $\Lambda_{t}=7.1 \mathrm{TeV}$ consistent with experiment.

To obtain the observed baryon asymmetry eq. (1.1) with a top source requires a large top-lepton interaction, with a fairly low scale $\Lambda_{\mathrm{QL}} \lesssim 1 \mathrm{TeV}$ (as always, precise statements cannot be made due to significant theoretical uncertainties). To properly describe such a set-up probably requires going beyond the EFT description and adding the light degree of freedom that mediates the interaction. This might change the quantitative results, but we expect the results to be qualitatively the same. As the strength of the new coupling increases, the interactions approach equilibrium, and a further increase has limited effect; this explains the asymptotic flattening of $Y_{B}$ for small cutoff $\Lambda_{\mathrm{QL}}$. The new coupling can 
boost the asymmetry by more than an order of magnitude. Naively one may have expected a $\mathcal{O}\left(10^{2}\right)$-increase, as the sphaleron washout reduces the chiral asymmetry in quarks by approximately this factor. However, even without the new interactions there is already transfer taking place via the SM Yukawa interactions. While the tau Yukawa is small, these effects already dominate the asymmetry for $\Lambda_{\mathrm{QL}} \rightarrow \infty$, as discussed in section 4.1.

While we considered here a top-tau interaction, the baryon asymmetry can be equally boosted via additional interactions between top quarks and electrons or muons as the lepton flavor is irrelevant for the baryon asymmetry. The asymmetry can also be boosted by inclusion of BSM couplings between top and one of the lighter quarks. Such couplings, if sufficiently strong, would also avoid efficient washout by strong sphalerons, as discussed in section 2.7 .

The effects of the dimension-six operator in eq. (2.23) in case of a lepton source is opposite as it now reduces the total asymmetry. As long as the new interactions are sufficiently suppressed $\Lambda_{\mathrm{QL}} \gtrsim 1 \mathrm{TeV}$, the chiral asymmetry stays mostly in the lepton sector and can be large enough to explain the observed value. However, for larger couplings the asymmetry can be suppressed by as much as two orders of magnitude. This effect can be seen in explicit models such as those studied in refs. [33, 34] where a lepton CPV source is considered in the context of a two-Higgs doublet model. The source term, however, must be made rather large compared to the tau source discussed in section 3 (about a factor hundred larger) to generate a baryon asymmetry consistent with observations. The source originates from CPV Yukawa interactions with heavier non-SM Higgs fields, which can be increased without running in conflict with experiments as long as the mixing angle between the heavier Higgs and the SM Higgs is sufficiently small. EDM bounds are also avoided by choosing couplings between the non-SM scalars and electrons and lighter quarks to be sufficiently small. The heavy scalars, however, lead to effective operators of the form of eq. (2.23) (although the actual lepton and quark flavor can vary) that, as shown in figure 6, suppress the baryon asymmetry. As such, larger CPV sources are required. This example shows that the rather simple framework considered here, based on effective operators can qualitatively describe the features of more complicated BSM models and can provide a useful guide in model building.

\section{Discussion and conclusions}

EWBG is an interesting framework for the generation of the matter-antimatter asymmetry. As EWBG takes place at a relatively low energy scale, the BSM physics required for the first-order phase transition and CPV can be tested in various experiments ranging from colliders and EDM searches to the detection of gravitational waves. EWBG however cannot take place within the SM and BSM physics is required to ensure a first-order electroweak phase transition and sufficient CPV.

Many EWBG models have been proposed and in this work we do not commit to a specific model but instead applied EFT methods to describe the dynamics of EWBG. However, ref. [16] showed that EWBG cannot be embedded into the SM-EFT framework as a first-order phase transition requires additional light degrees of freedom that cannot 
be integrated out of the theory. In this work, we therefore describe the phase transition with a phenomenological ansatz and describe the required CPV with effective dimensionsix operators containing SM fields only. Additional CPV operators involving the light degrees of freedom can certainly exist and be relevant, but as these are difficult to test experimentally, we leave them to future work.

We consider flavor-diagonal CPV dimension-six Yukawa operators of quarks and charged leptons with couplings that scale as $y_{f} / \Lambda_{f}^{2}$, where $\Lambda_{f}$ is the scale where new physics appears and the EFT description breaks down. The resulting CPV source term that appears in the transport equation and drives the eventual generation of the baryon asymmetry scales as $y_{f}^{2} / \Lambda_{f}^{2}$. As such, for the same value of $\Lambda_{f}$ it might be expected that the top quark would provide the largest baryon asymmetry. The recent electron EDM limit [25] sets a very strong constraint $\Lambda_{t} \geq 7.1 \mathrm{TeV}$ which ensures that a top CPV source as studied in this work cannot lead to the observed baryon asymmetry. For lighter fermions the CPV source term is suppressed by $\left(y_{f} / y_{t}\right)^{2}$, and even though the scale $\Lambda_{f}$ is not as constrained, one might expect these light fermions to lead to even smaller asymmetries. We have investigated this in detail in this work and conclude that the naive scaling is not correct. Our main findings can be summarized as

- Despite the Yukawa suppression, a CPV tau source leads to a baryon asymmetry of the same order of magnitude as induced by a top source for $\Lambda_{\tau}=\Lambda_{t}$. As EDM constraints on $\Lambda_{\tau}$ are much weaker, the tau source can produce the observed baryon asymmetry where a top source cannot. The relative effectiveness of the tau source with respect to the top source has several causes. While the top source is enhanced by $\left(y_{t} / y_{\tau}\right)^{2}$, the Yukawa and strong sphaleron rate effectively wash out a chiral asymmetry in top quarks. This washout is far less effective for tau leptons that have much smaller Yukawa couplings and do not participate in strong sphaleron interactions. In addition, a chiral asymmetry in leptons diffuses much further into the plasma and electroweak sphaleron processes have more time to convert the chiral asymmetry into a baron asymmetry.

- We performed analytical and numerical calculations of varying sophistication of the baryon asymmetry in case of a tau CPV source. The analytical and numerical results are found to be in very good agreement. The analytical solutions provide insight into the dependence of the baryon asymmetry on parameters related to the phase transition, such as the bubble-wall velocity, and the size of the lepton Yukawa coupling. Depending on several parameters, we can identify regions where the baryon asymmetry scales as $\propto y_{f}^{2}$, as naively expected, but also as $\propto y_{f}$ showing explicit deviations from the naive scaling. As such, lighter fermions can be relatively effective in generating a baryon asymmetry.

- While tau leptons are more efficient than might be expected, our analytical solution shows that baryon asymmetries induced by muon or electron CPV dimension-six Yukawa interactions are suppressed by respectively $\left(y_{\mu} / y_{\tau}\right)^{2}$ and $\left(y_{e} / y_{\tau}\right)^{2}$. Such 
sources therefore lead to much too small baryon asymmetries and only the tau source is still phenomenologically relevant.

- Even in case of a top CPV source, leptons play an important role. The SM Yukawa interactions can convert a chiral asymmetry in quarks to a chiral asymmetry in leptons. This conversion is proportional to the tau Yukawa coupling and therefore often neglected. We find, however, that including tau leptons explicitly in the transport equations leads to a significant enhancement of the total baryon asymmetry up to an order of magnitude. This enhancement is a general feature over a wide range of bubble-wall velocities. We conclude that EWBG scenarios with CPV in the quark sector should explicitly include leptons in the transport equations. Despite this enhancement, the CPV top source from the dimension-six Yukawa interactions consistent with EDM experiments leads to a too small baryon asymmetry. This effectively rules out the minimal EWBG scenario of ref. [24], although it must be stressed that the involved theoretical uncertainties are large.

- As a side result, we find that the fast-rate approximation which is often applied in studies of EWBG with CPV sources including top quarks, is not reliable. In our setup it significantly overestimates the total baryon asymmetry. This conclusion is in line with ref. [73]. We recommend to avoid its use and to instead solve the complete set of transport equations.

- While the washout of the chiral asymmetry for a bottom CPV source is less effective than for a top CPV source, this does not compensate for the $\left(y_{b} / y_{t}\right)^{2}$ suppression of the CPV source. We find that a CPV source from dimension-six bottom Yukawa interactions leads to a baryon asymmetry that is approximately two orders of magnitude smaller than a top CPV source for the same value $\Lambda_{b}=\Lambda_{t}$. For values of $\Lambda_{b}$ consistent with EDM experiments this leads to a too small baryon asymmetry. For dimension-six Yukawa couplings for even lighter quarks the induced baryon asymmetries are even smaller.

- The total baryon asymmetry can be significantly altered in BSM models with more effective chiral-symmetry-breaking interactions between quarks and leptons than are present in the SM. In this work, we have modeled such interactions with effective dimension-six top-tau interactions that can be induced in explicit BSM models by the exchange of new particles. Such interactions can enhance, in case of a CPV quark source, or decrease, in case of a CPV lepton source, the baryon asymmetry by orders of magnitude. This mechanism can be useful to guide model building.

As a final remark, we would like to emphasize that although we looked at a specific implementation of CP violation via dimension-six Yukawa operators, our qualitative conclusions are more general. The importance of leptons and the related mechanism to boost the baryon asymmetry by transferring the chiral asymmetry from the quark to the lepton sector (or suppress it by doing the opposite), are independent of the details of our set-up. 


\section{Acknowledgments}

MP and JvdV are supported by the Netherlands Organization for Scientific Research (NWO). We thank Graham White and Kaori Fuyuto for helpful discussions. JvdV thanks the Amherst Center for Fundamental Interactions and the University of Massachusetts, Amherst, for the hospitality during the completion of this work.

\section{A Benchmark values}

In this appendix we list the values of various parameters for our benchmark scenario. For the values of the coupling constants at the electroweak scale $\mu=m_{Z}$ we use

$$
g^{\prime}=0.36, \quad g=0.65, \quad g_{s}=1.23, \quad y_{t}=1, \quad y_{b}=0.02, \quad y_{\tau}=0.01
$$

The diffusion constants are taken from refs. [31, 63]

$$
D_{t} \simeq \frac{6}{T}, \quad D_{q} \simeq \frac{6}{T}, \quad D_{\tau} \simeq \frac{100}{T}, \quad D_{l} \simeq \frac{380}{T}, \quad D_{h} \simeq \frac{100}{T} .
$$

For the nucleation temperature we use

$$
T_{N}=88 \mathrm{GeV}
$$

The value of the Higgs field in the broken vacuum, the bubble-wall width and the bubblewall speed are given by:

$$
v_{N}=152 \mathrm{GeV}, \quad L_{w}=0.11 \mathrm{GeV}^{-1}, \quad v_{w}=0.05 .
$$

The interaction rates are the same as in $[16]^{4}$ their numerical values are listed in table 5 .

\section{B Weak sphalerons}

In this appendix we discuss the inclusion of the weak sphaleron rates in the transport equations eq. (2.13) and compare the results with the two-step procedure described in section 2.5. We use the subscript $L$ to denote left-handed, and $l$ for leptons.

The EW sphaleron transitions involve 3 left-handed quarks and one left-handed lepton per family, changing $\Delta N_{\mathrm{cs}}=\Delta B=\Delta L=n_{f}$. The rate at which the baryon density $n_{b}$ approaches equilibrium is given by the principle of detailed balance $[81,82]$

$$
\partial n_{b}=-n_{f} \Gamma_{\mathrm{ws}}\left(\mu_{\mathrm{ws}}+\mu_{\mathrm{ws}}^{0}\right)
$$

with

$$
\mu_{\mathrm{ws}}=\sum\left(3 \mu_{q_{L}}+\mu_{l_{L}}\right)=\sum\left(3 \frac{n_{q_{L}}}{k_{q_{L}}}+\frac{n_{l_{L}}}{k_{L}}\right) \simeq \frac{1}{2} \sum\left(n_{q_{L}}+n_{l_{L}}\right) \equiv \frac{1}{2} n_{L}
$$

\footnotetext{
${ }^{4}$ The relaxation rate $\Gamma_{M}$ corresponds to $\Gamma_{M}^{-}$in [16]. The rate $\Gamma_{M}^{+}$has been set to zero, which is a good approximation.
} 


\begin{tabular}{|l|c|c|}
\hline & Broken phase & Symmetric phase \\
\hline$\Gamma_{\mathrm{ss}}$ & 0.26 & 0.26 \\
\hline$\Gamma_{M}^{(t)}$ & 104 & 0 \\
\hline$\Gamma_{Y}^{(t)}$ & 2.7 & 2.7 \\
\hline$\Gamma_{M}^{(b)}$ & $3.7 \cdot 10^{-2}$ & 0 \\
\hline$\Gamma_{Y}^{(b)}$ & $1.1 \cdot 10^{-3}$ & $1.1 \cdot 10^{-3}$ \\
\hline$\Gamma_{M}^{(l)}$ & $4.6 \cdot 10^{-2}$ & 0 \\
\hline$\Gamma_{Y}^{(l)}$ & $1.0 \cdot 10^{-4}$ & $1.0 \cdot 10^{-4}$ \\
\hline$\Gamma_{\mathrm{ws}}$ & 0 & $4.7 \cdot 10^{-4}$ \\
\hline
\end{tabular}

Table 5. Asymptotic values of the interaction rates in the broken and symmetric phase. All rates are in $\mathrm{GeV}$. The relaxation rates $\Gamma_{M}^{(f)}$ are a function of the bounce solution $\phi_{b}$ and vary over the bubble wall.

with $n_{i}=k_{i} \mu_{i}$ the relation between the number density and the (rescaled) chemical potential, and with $n_{L}$ the chiral density. The summation is over families, and we approximated the $k_{i}$-functions with their zero-temperature values.

The $\mu^{0}$-term takes into account the initial conditions. In the transport equations eq. (2.13) the initial densities are zero before the passage of the bubble wall and such terms are absent.

\section{B.1 Two-step approach}

Electroweak sphalerons convert the chiral asymmetry into a baryon asymmetry. If the corresponding rate is slower than the other interaction rates it decouples from the transport equations, and we can model it as a two-step process. The outcome of the transport equations for the fast processes is used as initial condition for the sphaleron equation, that is $\mu_{\mathrm{ws}}^{0}=\frac{1}{2} n_{L}^{0}$.

The strong sphalerons are fast on the relevant time-scale and are assumed to be in equilibrium, which sets $\mu_{\mathrm{ss}}=\sum\left(n_{q_{L}}-n_{u_{R}}-n_{d_{R}}\right)=0$. Physically, this means that any left-handed quarks produced by sphalerons are instantly divided over left- and right-handed quarks of the same generation via strong sphaleron transitions.

The baryon asymmetry is relaxed by the $\mu_{\mathrm{ws}}$-term in eq. (B.1), which we want to rewrite in terms of $n_{b}$ for the equation to be closed. The quark part we rewrite using

$$
n_{b}=n_{b}^{L}+n_{b}^{R}=2 n_{b}^{L}=\frac{2}{3} \sum n_{q_{L}} .
$$

For the leptons we use that weak sphalerons conserve $B-L$. If the CPV source is in the quark sector, and the weak lepton Yukawa interactions are neglected, then leptons are only produced via weak sphalerons and the right-handed lepton densities are negligible. Then

$$
n_{b}=n_{l}=n_{l}^{L}+n_{l}^{R} \simeq n_{l}^{L}=\sum n_{l_{L}} .
$$


It would seem that this expression no longer holds if the source is in the lepton sector, or more generally, if lepton interactions are included, as it is then no longer a good approximation to neglect $\tau_{R}$. However, neglecting the difference in diffusion constants for the left- and right-handed leptons, both chiralities are produced in equal amounts by the Yukawa/relaxation/source interactions and their local densities cancel. The net lepton density then still arises from weak sphaleron transitions as assumed in eq. (B.4).

The chemical potential can be rewritten

$$
\mu_{\mathrm{ws}}=\frac{1}{2} \sum\left(n_{q_{L}}+n_{l_{L}}\right)=\frac{1}{2}\left(\frac{3}{2}+1\right) n_{b} \equiv \frac{1}{3} \mathcal{R} n_{b},
$$

with $\mathcal{R}=15 / 4$. Putting it all together then gives for the baryon asymmetry

$$
\partial n_{b}=-\Gamma_{\mathrm{ws}}\left(\frac{3}{2} n_{L}^{0}+\mathcal{R} n_{b}\right)
$$

with $\partial n_{b}=v_{w} n_{b}^{\prime}-D_{q} n_{b}^{\prime \prime}$.

Inside the broken phase $n_{b}$ is constant, as there are no sphaleron transitions. The broken phase becomes our universe, and this solution is what we are after. Normalizing $Y_{B}(-\infty)=0$ then gives eq. (2.17).

\section{B.2 One-step approach}

In the one-step approach we include the weak sphalerons in the transport equations, to calculate the baryon asymmetry in one go. In this case we can set $\mu_{\mathrm{ws}}^{0}=0$, as the initial densities are zero. Only left-handed particles are produced by the weak sphalerons, and their transport equation picks up an extra term. For the third generation the transport eq. (2.13) is changed to

$$
\begin{aligned}
\partial_{\mu} q^{\mu} & =+\Gamma_{M}^{(t)} \mu_{M}^{(t)}+\Gamma_{M}^{(b)} \mu_{M}^{(b)}+\Gamma_{Y}^{(t)} \mu_{Y}^{(t)}+\Gamma_{Y}^{(b)} \mu_{Y}^{(b)}-2 \Gamma_{\mathrm{ss}} \mu_{\mathrm{ss}}-3 \Gamma_{\mathrm{ws}} \mu_{\mathrm{ws}} \\
\partial_{\mu} l^{\mu} & =+\Gamma_{M}^{(\tau)} \mu_{M}^{(\tau)}+\Gamma_{Y}^{(\tau)} \mu_{Y}^{(\tau)}-\Gamma_{\mathrm{ws}} \mu_{\mathrm{ws}}-S_{\tau}
\end{aligned}
$$

where for simplicity we have considered CPV in the tau sector only.

To solve the set of transport equations including the weak sphaleron rate we make some approximations. First, we neglect bottom and charm Yukawa interactions. Second, as we have seen in the two-step description, it is a good approximation to neglect the Higgs density and set $h \simeq 0$. Third, the strong sphaleron and top Yukawa interactions are fast on the scale of the relevant leptonic interactions, and can be assumed in equilibrium, which gives $q \simeq t+b$ and $\frac{4}{3} t \simeq \frac{2}{3} q$, where we have approximated the $k_{i}$ functions with their zero-temperature values. We define the new fields

$$
\bar{q}=\frac{1}{2}(q+t+b), \quad \bar{l}=\frac{1}{2}(l-\tau), \quad \delta l=l+\tau .
$$

The usefulness of $\delta l$ is that it gives the net lepton number produced by weak sphalerons which vanishes in the limit of lepton number conservation; in the two-step process we only 
had the $\bar{l}$ equation as $\delta l=0$ and the baryons were decoupled. The transport equations in terms of the new variables become

$$
\begin{aligned}
\partial_{\mu} \bar{l}^{\mu} & =+\Gamma_{M}^{(\tau)} \mu_{M}^{(\tau)}+\Gamma_{Y}^{(\tau)} \mu_{Y}^{(\tau)}-\frac{1}{2} \Gamma_{\mathrm{ws}} \mu_{\mathrm{ws}}-S_{\tau}, \\
\partial_{\mu} \delta l^{\mu} & =-\Gamma_{\mathrm{ws}} \mu_{\mathrm{ws}}, \\
\partial_{\mu} \bar{q}^{\mu} & =-\frac{3}{2} \Gamma_{\mathrm{ws}} \mu_{\mathrm{ws}} .
\end{aligned}
$$

These equations ensure that any left-handed quark number density produced by the weak sphalerons is immediately evenly spread among left- and right-handed quarks by the strong sphalerons. This explains the factor $1 / 2$ in the $\bar{q}$ equation (which is the averaged sum over left- and right-handed quarks) as compared to the coefficient of the weak sphalerons rate in the $q$-equation in eq. (B.7).

We can further use that sphalerons conserve $B-L$ which gives the additional relation

$$
n_{b}=n_{l} \quad \Rightarrow \quad \delta l=\frac{2}{3} \bar{q}
$$

which we use to eliminate the $\delta l$ equation. Using $n_{b}=2 \bar{q}$ then finally gives

$$
\begin{aligned}
\partial_{\mu} \bar{l}^{\mu} & =+\Gamma_{M}^{(\tau)} \mu_{M}^{(\tau)}+\Gamma_{Y}^{(\tau)} \mu_{Y}^{(\tau)}-\frac{1}{2} \Gamma_{\mathrm{ws}} \mu_{\mathrm{ws}}-S_{\tau}, \\
\partial_{\mu} n_{b}^{\mu} & =-3 \Gamma_{\mathrm{ws}} \mu_{\mathrm{ws}} .
\end{aligned}
$$

The chemical potentials in terms of these variables are

$$
\begin{aligned}
\mu_{Y}^{(\tau)} \simeq \mu_{M}^{(\tau)} & =\left(\frac{\tau}{k_{\tau}}-\frac{l}{k_{l}}\right)=\left(-\frac{k_{l}+k_{\tau}}{k_{l} k_{\tau}} \bar{l}+\frac{k_{l}-k_{\tau}}{6 k_{l} k_{\tau}} n_{b}\right), \\
\mu_{\mathrm{ws}} & =\frac{1}{2} \sum\left(q_{L}+l_{L}\right)=\frac{1}{2}(3 \bar{q}+2 \delta l+l)=\left(\frac{\bar{l}}{2}+\frac{7}{6} n_{b}\right),
\end{aligned}
$$

where we used $l+\tau=\delta l=\frac{1}{3} n_{b}$ and $l-\tau=2 \bar{l}$. The baryon asymmetry is the constant value in the broken phase, and the end result is $Y_{B}=n_{b}(z>0) / s$.

We have calculated the baryon asymmetry $Y_{B}$ for a lepton source for our benchmark parameters, and more general as a function of the bubble-wall velocity. Over the whole range the difference between the one- and two-step calculation is at most a few percent. We conclude that the two-step procedure is an accurate approximation, even in the regime where $\Gamma_{\mathrm{ws}} \gtrsim \Gamma_{y}^{(l)}$ and there is no clear separation of length scales.

\section{Numerical method}

In this appendix we describe our method to solve the numerical transport equations in eq. (2.13). The transport equations are of the form

$$
v_{w} \vec{x}^{\prime}-D \vec{x}^{\prime \prime}+b \vec{x}+\vec{s}=0
$$

with $\vec{x}=(q, t, \ldots)^{T}$ the number densities, $v_{w}=v_{w} \mathbb{1}, D=\operatorname{diag}\left(D_{q}, D_{t}, \ldots\right), b$ a matrix that contains the rates which can be read from the transport equations, and $\vec{s}$ the source vector. 
We denote the number of equations, that is the dimension of $\vec{x}$, by $n_{F}$. These equations are solved with the boundary conditions that the number densities vanish far away from the bubble wall $\lim _{z= \pm \infty} \vec{x}=0 .{ }^{5}$

In the asymptotic regions $z<z_{-}<0$ and $z>z_{+}>0$ the source vanishes and the rates become constant $\vec{s}=0$ and $b=$ const., and we can solve the equations analytically. We consider first the $z<z_{-}$region of the symmetric phase. For the asymptotic solution $\vec{x}_{-}$we use the Ansatz

$$
x_{i}=A_{i} \mathrm{e}^{\alpha z}, \quad i=1, \ldots, n_{F},
$$

and substitute in the asymptotic form of the transport equations. Setting $A_{1}=1$, we can solve the system numerically for $A_{2}, \ldots, A_{n_{F}}$ and $\alpha$. This gives $2 n_{F}$ solutions from which we select the $n_{F}$ decaying solutions, that is the solutions with positive $\alpha_{j}\left(\alpha_{j}>0\right.$ for $\left.j=1, \ldots, n_{F}\right)$. The solution in the asymptotic symmetric phase is then

$$
x_{i}=\sum_{j=1}^{n_{F}} a_{j} A_{i}\left(\alpha_{j}\right) \mathrm{e}^{\alpha_{j} z}, \quad z<z_{-},
$$

with $a_{j}$ normalization constants. Likewise the equations can be solved in the asymptotic region $z>z_{+}$in the broken phase (now with the asymptotic $b$-values of the broken phase). We denote the parameters in the broken phase by an overbar. The asymptotic solution is ${ }^{6}$

$$
x_{i}=\sum_{j=1}^{n_{F}} \bar{a}_{j} \bar{A}_{i}\left(\bar{\alpha}_{j}\right) \mathrm{e}^{-\bar{\alpha}_{j} z}, \quad z>z_{+},
$$

with $\bar{\alpha}_{j}>0$.

The idea now is to solve the transport equations and match to the asymptotic solutions. That is, we solve the equations with boundary conditions set at $z_{ \pm}$. Eq. (C.2) gives $2 n_{F}$ boundary conditions $\vec{x}\left(z_{-}\right), \vec{x}^{\prime}\left(z_{-}\right)$at $z_{-}$in terms of $n_{F}$ normalization constants $a_{j}$, and eq. (C.3) gives $2 n_{F}$ boundary conditions $\vec{x}\left(z_{+}\right), \vec{x}^{\prime}\left(z_{+}\right)$at $z_{+}$in terms of $n_{F}$ normalization constants $\bar{a}_{j}$. The total of $2 n_{F}$ normalization constants are determined by a shooting method.

To be more specific, we start with the analytical solution as a boundary condition at $z_{-}$, we pick the $n_{F}$ normalization constants $a_{j}$, and numerically evolve to $z=0$ using the full transport equations eq. (C.1). Call this solution $\vec{x}\left(0_{-}\right) \equiv \vec{x}_{-}\left(a_{j}\right)$. Likewise we pick values for $\bar{a}_{j}$, and numerically evolve the solution at $z_{+}$back to $z=0$, and call this solution $\vec{x}\left(0_{+}\right) \equiv \vec{x}_{+}\left(\bar{a}_{j}\right)$. We adjust $a_{j}, \bar{a}_{k}$ via a shooting procedure such that at $z=0$ the first and second derivative of the two solutions match, i.e.

$$
\vec{x}_{-}\left(a_{j}\right)=\vec{x}_{+}\left(\bar{a}_{j}\right), \quad \vec{x}_{-}^{\prime}\left(a_{j}\right)=\vec{x}_{+}^{\prime}\left(\bar{a}_{j}\right) .
$$

\footnotetext{
${ }^{5}$ Including both the tau and lepton equation, or including the weak sphalerons in a one-step process, this boundary condition has to be generalized to $\lim _{z= \pm \infty} \vec{x}=$ const., as some combination of number densities do not have interactions in the broken phase, and therefore do not necessary relax to zero.

${ }^{6}$ This asymptotic solution differs from that used in the semi-analytical method of [73]. The difference is negligible if the boundary conditions are set at $z_{+}$large enough (compared to the relevant length scales), such that the non-decaying terms in the semi-analytical solution are also negligible. This can always be done in the planar wall approximation for the bubble profile used in this paper, but not neccessarily for the actual profile of a finite-sized bubble.
} 
The shooting procedure can be implemented using Newton's method by starting with initial guesses for $a_{j}, \bar{a}_{i}$ and then calculating $x_{-}, x_{+}$. As a measure of goodness of the solution we define the function

$$
\vec{F}=\left(\begin{array}{l}
\vec{x}_{-}-\vec{x}_{+} \\
\vec{x}_{-}^{\prime}-\vec{x}_{+}^{\prime}
\end{array}\right) .
$$

Newton's method gives a way to find the next values of $\vec{b}=\left(\begin{array}{l}\vec{a} \\ \vec{a}\end{array}\right)$, that minimize the $\vec{F}$ function (both $\vec{b}$ and $\vec{F}$ are $2 n_{F}$ dimensional vectors)

$$
\vec{b}_{\text {new }}=\vec{b}-J(\vec{b})^{-1} \vec{F}(\vec{b}), \quad \text { with } \quad J=\left[\begin{array}{cccc}
\frac{\partial F_{1}}{\partial b_{1}} & \frac{\partial F_{1}}{\partial b_{2}} & \cdots & \frac{\partial F_{1}}{\partial b_{2 n_{F}}} \\
\frac{\partial F_{2}}{\partial b_{1}} & \frac{\partial F_{2}}{\partial b_{2}} & \cdots & \frac{\partial F_{2}}{\partial b_{2 n_{F}}} \\
\vdots & \cdots & \cdots & \vdots \\
\frac{\partial F_{2 n_{F}}}{\partial b_{1}} & \frac{\partial F_{2 n_{F}}}{\partial b_{2}} & \cdots & \frac{\partial F_{2 n_{F}}}{\partial b_{2 n_{F}}}
\end{array}\right] .
$$

We reiterate the process until $\sum_{i}^{2 n_{F}}\left|F_{i}\right|<\epsilon$ smaller than the required precision. We choose $\epsilon=10^{-6}$, and have checked that the result does not change for smaller values of $\epsilon$.

We calculate the entries of the Jacobian as follows. We choose $\tilde{b}=\left(b_{1}, \ldots, b_{i}(1+\right.$ $\left.\epsilon), \ldots, b_{2 n_{F}}\right)$ and calculate with these entries $\left(\tilde{x}_{-}, \tilde{x}_{+}\right)$and determine $\tilde{F}_{j}$. Then $\partial F_{j} / \partial b_{i}=$ $\left(\tilde{F}_{j}-F_{j}\right) /\left(b_{i} \epsilon\right)$. We do this for $i=1,2, \ldots, 2 n_{F}$ to find all entries of the Jacobian.

Open Access. This article is distributed under the terms of the Creative Commons Attribution License (CC-BY 4.0), which permits any use, distribution and reproduction in any medium, provided the original author(s) and source are credited.

\section{References}

[1] Planck collaboration, Planck 2015 results. XIII. Cosmological parameters, Astron. Astrophys. 594 (2016) A13 [arXiv:1502.01589] [INSPIRE].

[2] A.D. Sakharov, Violation of CP invariance, C asymmetry and baryon asymmetry of the universe, Pisma Zh. Eksp. Teor. Fiz. 5 (1967) 32 [Usp. Fiz. Nauk 161 (1991) 61] [InSPIRE].

[3] A. Arhrib, P.M. Ferreira and R. Santos, Are there hidden scalars in LHC Higgs results?, JHEP 03 (2014) 053 [arXiv: 1311.1520] [INSPIRE].

[4] C.-Y. Chen, S. Dawson and M. Sher, Heavy Higgs searches and constraints on two Higgs doublet models, Phys. Rev. D 88 (2013) 015018 [Erratum ibid. D 88 (2013) 039901] [arXiv: 1305.1624] [INSPIRE].

[5] W.-F. Chang, T. Modak and J.N. Ng, Signal for a light singlet scalar at the LHC, Phys. Rev. D 97 (2018) 055020 [arXiv:1711.05722] [INSPIRE].

[6] CMS collaboration, A search for beyond Standard Model light bosons decaying into muon pairs, CMS-PAS-HIG-16-035, CERN, Geneva, Switzerland (2016).

[7] C. Englert et al., Precision measurements of Higgs couplings: implications for new physics scales, J. Phys. G 41 (2014) 113001 [arXiv:1403.7191] [INSPIRE].

[8] I. Brivio and M. Trott, The Standard Model as an effective field theory, Phys. Rept. 793 (2019) 1 [arXiv: 1706.08945] [INSPIRE]. 
[9] T. Han and Y. Li, Genuine CP-odd observables at the LHC, Phys. Lett. B 683 (2010) 278 [arXiv:0911.2933] [INSPIRE].

[10] F. Boudjema, R.M. Godbole, D. Guadagnoli and K.A. Mohan, Lab-frame observables for probing the top-Higgs interaction, Phys. Rev. D 92 (2015) 015019 [arXiv:1501.03157] [INSPIRE].

[11] J. Ellis, Discrete glimpses of the physics landscape after the Higgs discovery, J. Phys. Conf. Ser. 631 (2015) 012001 [arXiv: 1501. 05418] [INSPIRE].

[12] A. Askew, P. Jaiswal, T. Okui, H.B. Prosper and N. Sato, Prospect for measuring the CP phase in the $h \tau \tau$ coupling at the LHC, Phys. Rev. D 91 (2015) 075014 [arXiv:1501.03156] [INSPIRE].

[13] F. Demartin, F. Maltoni, K. Mawatari and M. Zaro, Higgs production in association with a single top quark at the LHC, Eur. Phys. J. C 75 (2015) 267 [arXiv:1504.00611] [InSPIRE].

[14] T. Chupp, P. Fierlinger, M. Ramsey-Musolf and J. Singh, Electric dipole moments of atoms, molecules, nuclei and particles, Rev. Mod. Phys. 91 (2019) 015001 [arXiv:1710. 02504] [INSPIRE].

[15] C. Balázs, G. White and J. Yue, Effective field theory, electric dipole moments and electroweak baryogenesis, JHEP 03 (2017) 030 [arXiv: 1612.01270] [INSPIRE].

[16] J. de Vries, M. Postma, J. van de Vis and G. White, Electroweak baryogenesis and the Standard Model effective field theory, JHEP 01 (2018) 089 [arXiv:1710.04061] [INSPIRE].

[17] LISA collaboration, Laser Interferometer Space Antenna, arXiv:1702.00786 [INSPIRE].

[18] C. Caprini et al., Science with the space-based interferometer eLISA. II: gravitational waves from cosmological phase transitions, JCAP 04 (2016) 001 [arXiv: 1512.06239] [INSPIRE].

[19] D.E. Morrissey and M.J. Ramsey-Musolf, Electroweak baryogenesis, New J. Phys. 14 (2012) 125003 [arXiv: 1206. 2942] [INSPIRE].

[20] J.M. Cline, Baryogenesis, in Les Houches Summer School - Session 86: particle physics and cosmology. The fabric of spacetime, Les Houches, France, 31 July-25 August 2006 [hep-ph/0609145] [INSPIRE].

[21] M. Trodden, Electroweak baryogenesis, Rev. Mod. Phys. 71 (1999) 1463 [hep-ph/9803479] [INSPIRE].

[22] G.A. White, A pedagogical introduction to electroweak baryogenesis, IOP Publishing, U.K. (2016) [INSPIRE].

[23] V. Vaskonen, Electroweak baryogenesis and gravitational waves from a real scalar singlet, Phys. Rev. D 95 (2017) 123515 [arXiv:1611.02073] [INSPIRE].

[24] S.J. Huber, M. Pospelov and A. Ritz, Electric dipole moment constraints on minimal electroweak baryogenesis, Phys. Rev. D 75 (2007) 036006 [hep-ph/0610003] [INSPIRE].

[25] ACME collaboration, Improved limit on the electric dipole moment of the electron, Nature 562 (2018) 355 [INSPIRE].

[26] M. Joyce, T. Prokopec and N. Turok, Efficient electroweak baryogenesis from lepton transport, Phys. Lett. B 338 (1994) 269 [hep-ph/9401352] [INSPIRE].

[27] V. Cirigliano, W. Dekens, J. de Vries and E. Mereghetti, Constraining the top-Higgs sector of the Standard Model effective field theory, Phys. Rev. D 94 (2016) 034031 [arXiv: 1605.04311] [INSPIRE]. 
[28] G.F. Giudice and M.E. Shaposhnikov, Strong sphalerons and electroweak baryogenesis, Phys. Lett. B 326 (1994) 118 [hep-ph/9311367] [INSPIRE].

[29] S. Tulin and P. Winslow, Anomalous B meson mixing and baryogenesis, Phys. Rev. D 84 (2011) 034013 [arXiv: 1105. 2848] [INSPIRE].

[30] J. Brod, U. Haisch and J. Zupan, Constraints on CP-violating Higgs couplings to the third generation, JHEP 11 (2013) 180 [arXiv:1310.1385] [INSPIRE].

[31] M. Joyce, T. Prokopec and N. Turok, Nonlocal electroweak baryogenesis. Part 1: thin wall regime, Phys. Rev. D 53 (1996) 2930 [hep-ph/9410281] [INSPIRE].

[32] D.J.H. Chung, B. Garbrecht, M.J. Ramsey-Musolf and S. Tulin, Lepton-mediated electroweak baryogenesis, Phys. Rev. D 81 (2010) 063506 [arXiv:0905.4509] [INSPIRE].

[33] C.-W. Chiang, K. Fuyuto and E. Senaha, Electroweak baryogenesis with lepton flavor violation, Phys. Lett. B 762 (2016) 315 [arXiv:1607.07316] [InSPIRE].

[34] H.-K. Guo, Y.-Y. Li, T. Liu, M. Ramsey-Musolf and J. Shu, Lepton-flavored electroweak baryogenesis, Phys. Rev. D 96 (2017) 115034 [arXiv:1609.09849] [InSPIRE].

[35] M. Gurtler, E.-M. Ilgenfritz and A. Schiller, Where the electroweak phase transition ends, Phys. Rev. D 56 (1997) 3888 [hep-lat/9704013] [INSPIRE].

[36] M. Laine and K. Rummukainen, What's new with the electroweak phase transition?, Nucl. Phys. Proc. Suppl. 73 (1999) 180 [hep-lat/9809045] [INSPIRE].

[37] Y. Aoki, F. Csikor, Z. Fodor and A. Ukawa, The endpoint of the first order phase transition of the $\mathrm{SU}(2)$ gauge Higgs model on a four-dimensional isotropic lattice, Phys. Rev. D 60 (1999) 013001 [hep-lat/9901021] [INSPIRE].

[38] F. Csikor, Z. Fodor and J. Heitger, Endpoint of the hot electroweak phase transition, Phys. Rev. Lett. 82 (1999) 21 [hep-ph/9809291] [INSPIRE].

[39] J.R. Espinosa and M. Quirós, The electroweak phase transition with a singlet, Phys. Lett. B 305 (1993) 98 [hep-ph/9301285] [INSPIRE].

[40] J.R. Espinosa and M. Quirós, Novel effects in electroweak breaking from a hidden sector, Phys. Rev. D 76 (2007) 076004 [hep-ph/0701145] [INSPIRE].

[41] V. Barger, P. Langacker, M. McCaskey, M.J. Ramsey-Musolf and G. Shaughnessy, LHC phenomenology of an extended Standard Model with a real scalar singlet, Phys. Rev. D 77 (2008) 035005 [arXiv:0706.4311] [INSPIRE].

[42] J.R. Espinosa, T. Konstandin, J.M. No and M. Quirós, Some cosmological implications of hidden sectors, Phys. Rev. D 78 (2008) 123528 [arXiv:0809.3215] [INSPIRE].

[43] J.R. Espinosa, T. Konstandin and F. Riva, Strong electroweak phase transitions in the Standard Model with a singlet, Nucl. Phys. B 854 (2012) 592 [arXiv:1107.5441] [InSPIRE].

[44] J.M. Cline and K. Kainulainen, Electroweak baryogenesis and dark matter from a singlet Higgs, JCAP 01 (2013) 012 [arXiv:1210.4196] [INSPIRE].

[45] A.I. Bochkarev, S.V. Kuzmin and M.E. Shaposhnikov, Electroweak baryogenesis and the Higgs boson mass problem, Phys. Lett. B 244 (1990) 275 [INSPIRE].

[46] N. Turok and J. Zadrozny, Phase transitions in the two doublet model, Nucl. Phys. B 369 (1992) 729 [INSPIRE]. 
[47] A.T. Davies, C.D. froggatt, G. Jenkins and R.G. Moorhouse, Baryogenesis constraints on two Higgs doublet models, Phys. Lett. B 336 (1994) 464 [INSPIRE].

[48] J.M. Cline and P.-A. Lemieux, Electroweak phase transition in two Higgs doublet models, Phys. Rev. D 55 (1997) 3873 [hep-ph/9609240] [INSPIRE].

[49] J.M. Cline, K. Kainulainen and M. Trott, Electroweak baryogenesis in two Higgs doublet models and B meson anomalies, JHEP 11 (2011) 089 [arXiv:1107.3559] [INSPIRE].

[50] G.C. Dorsch, S.J. Huber, T. Konstandin and J.M. No, A second Higgs doublet in the early universe: baryogenesis and gravitational waves, JCAP 05 (2017) 052 [arXiv:1611.05874] [INSPIRE].

[51] J.O. Andersen et al., Nonperturbative analysis of the electroweak phase transition in the two Higgs doublet model, Phys. Rev. Lett. 121 (2018) 191802 [arXiv:1711.09849] [INSPIRE].

[52] T. Gorda, A. Helset, L. Niemi, T.V.I. Tenkanen and D.J. Weir, Three-dimensional effective theories for the two Higgs doublet model at high temperature, JHEP 02 (2019) 081 [arXiv: 1802.05056] [INSPIRE].

[53] P.H. Damgaard, A. Haarr, D. O'Connell and A. Tranberg, Effective field theory and electroweak baryogenesis in the singlet-extended Standard Model, JHEP 02 (2016) 107 [arXiv: 1512.01963] [INSPIRE].

[54] S.R. Coleman, The fate of the false vacuum. 1. Semiclassical theory, Phys. Rev. D 15 (1977) 2929 [Erratum ibid. D 16 (1977) 1248] [INSPIRE].

[55] P. John, Bubble wall profiles with more than one scalar field: a numerical approach, Phys. Lett. B 452 (1999) 221 [hep-ph/9810499] [INSPIRE].

[56] M.B. Gavela, P. Hernández, J. Orloff and O. Pene, Standard Model CP-violation and baryon asymmetry, Mod. Phys. Lett. A 9 (1994) 795 [hep-ph/9312215] [INSPIRE].

[57] M.B. Gavela, P. Hernández, J. Orloff, O. Pene and C. Quimbay, Standard Model CP-violation and baryon asymmetry. Part 2: finite temperature, Nucl. Phys. B 430 (1994) 382 [hep-ph/9406289] [INSPIRE].

[58] P. Huet and E. Sather, Electroweak baryogenesis and Standard Model CP-violation, Phys. Rev. D 51 (1995) 379 [hep-ph/9404302] [INSPIRE].

[59] T. Brauner, O. Taanila, A. Tranberg and A. Vuorinen, Computing the temperature dependence of effective CP-violation in the Standard Model, JHEP 11 (2012) 076 [arXiv: 1208.5609] [INSPIRE].

[60] C. Lee, V. Cirigliano and M.J. Ramsey-Musolf, Resonant relaxation in electroweak baryogenesis, Phys. Rev. D 71 (2005) 075010 [hep-ph/0412354] [INSPIRE].

[61] J.M. Cline, Is electroweak baryogenesis dead?, Phil. Trans. Roy. Soc. Lond. A 376 (2018) 20170116 [arXiv: 1704.08911] [INSPIRE].

[62] M. Joyce, T. Prokopec and N. Turok, Electroweak baryogenesis from a classical force, Phys. Rev. Lett. 75 (1995) 1695 [Erratum ibid. 75 (1995) 3375] [hep-ph/9408339] [INSPIRE].

[63] J.M. Cline, M. Joyce and K. Kainulainen, Supersymmetric electroweak baryogenesis, JHEP 07 (2000) 018 [hep-ph/0006119] [INSPIRE].

[64] Y.T. Chien, V. Cirigliano, W. Dekens, J. de Vries and E. Mereghetti, Direct and indirect constraints on CP-violating Higgs-quark and Higgs-gluon interactions, JHEP 02 (2016) 011 [arXiv: 1510.00725] [INSPIRE]. 
[65] J. Brod and E. Stamou, Electric dipole moment constraints on CP-violating heavy-quark Yukawas at next-to-leading order, arXiv:1810.12303 [INSPIRE].

[66] Belle collaboration, Search for the electric dipole moment of the $\tau$ lepton, Phys. Lett. B 551 (2003) 16 [hep-ex/0210066] [INSPIRE].

[67] W. Altmannshofer, J. Brod and M. Schmaltz, Experimental constraints on the coupling of the Higgs boson to electrons, JHEP 05 (2015) 125 [arXiv: 1503.04830] [INSPIRE].

[68] J. Brod and D. Skodras, Electric dipole moment constraints on CP-violating light-quark Yukawas, JHEP 01 (2019) 233 [arXiv:1811.05480] [INSPIRE].

[69] ATLAS collaboration, Cross-section measurements of the Higgs boson decaying to a pair of $\tau$ leptons in proton-proton collisions at $\sqrt{s}=13 \mathrm{TeV}$ with the ATLAS detector, ATLAS-CONF-2018-021, CERN, Geneva, Switzerland (2018).

[70] D.J.H. Chung, B. Garbrecht, M. Ramsey-Musolf and S. Tulin, Supergauge interactions and electroweak baryogenesis, JHEP 12 (2009) 067 [arXiv:0908.2187] [INSPIRE].

[71] M. Carena, M. Quirós, M. Seco and C.E.M. Wagner, Improved results in supersymmetric electroweak baryogenesis, Nucl. Phys. B 650 (2003) 24 [hep-ph/0208043] [InSPIRE].

[72] A.G. Cohen, D.B. Kaplan and A.E. Nelson, Diffusion enhances spontaneous electroweak baryogenesis, Phys. Lett. B 336 (1994) 41 [hep-ph/9406345] [INSPIRE].

[73] G.A. White, General analytic methods for solving coupled transport equations: from cosmology to beyond, Phys. Rev. D 93 (2016) 043504 [arXiv:1510.03901] [INSPIRE].

[74] D. Bödeker, G.D. Moore and K. Rummukainen, Chern-Simons number diffusion and hard thermal loops on the lattice, Phys. Rev. D 61 (2000) 056003 [hep-ph/9907545] [INSPIRE].

[75] G.D. Moore and K. Rummukainen, Classical sphaleron rate on fine lattices, Phys. Rev. D 61 (2000) 105008 [hep-ph/9906259] [INSPIRE].

[76] G.D. Moore, Sphaleron rate in the symmetric electroweak phase, Phys. Rev. D 62 (2000) 085011 [hep-ph/0001216] [INSPIRE].

[77] P. Huet and A.E. Nelson, Electroweak baryogenesis in supersymmetric models, Phys. Rev. D 53 (1996) 4578 [hep-ph/9506477] [INSPIRE].

[78] D.J.H. Chung, B. Garbrecht, M.J. Ramsey-Musolf and S. Tulin, Yukawa interactions and supersymmetric electroweak baryogenesis, Phys. Rev. Lett. 102 (2009) 061301 [arXiv:0808.1144] [INSPIRE].

[79] L. Fromme and S.J. Huber, Top transport in electroweak baryogenesis, JHEP 03 (2007) 049 [hep-ph/0604159] [INSPIRE].

[80] F.P. Huang, P.-H. Gu, P.-F. Yin, Z.-H. Yu and X. Zhang, Testing the electroweak phase transition and electroweak baryogenesis at the LHC and a circular electron-positron collider, Phys. Rev. D 93 (2016) 103515 [arXiv:1511.03969] [InSPIRE].

[81] M. Joyce, T. Prokopec and N. Turok, Constraints and transport in electroweak baryogenesis, Phys. Lett. B 339 (1994) 312 [hep-ph/9401351] [INSPIRE].

[82] A. Riotto and M. Trodden, Recent progress in baryogenesis, Ann. Rev. Nucl. Part. Sci. 49 (1999) 35 [hep-ph/9901362] [INSPIRE]. 